\title{
Maternal Gut Microbes Contribute to Rapid Acquisition of Intestinal Microbiota and High- Altitude Adaptation of Pre-Weaning Yak Calves
}

Jianbo Zhang

Chinese Academy of Agricultural Sciences Lanzhou Institute of Husbandry and Pharmaceutical

Sciences https://orcid.org/0000-0002-0478-2164

Zeyi Liang

CAAS LIHPS: Chinese Academy of Agricultural Sciences Lanzhou Institute of Husbandry and

Pharmaceutical Sciences

\section{Renqing Ding Kao}

Gannan Institute of Animal Husbandry Science

Jianlin Han

International Livestock Research Institute of Animal Science

Mei Du

CAAS LIHPS: Chinese Academy of Agricultural Sciences Lanzhou Institute of Husbandry and

Pharmaceutical Sciences

Anum Ali Ahmad

Lanzhou University School Of Life Sciences

Shengyi Wang

CAAS LIHPS: Chinese Academy of Agricultural Sciences Lanzhou Institute of Husbandry and Pharmaceutical Sciences

Ghasem Hosseini Salekdeh

Agricultural Biotechnology Research Institute of Iran

Ruijun Long

Lanzhou University School Of Life Sciences

\section{Ping Yan}

CAAS LIHPS: Chinese Academy of Agricultural Sciences Lanzhou Institute of Husbandry and Pharmaceutical Sciences

Xuezhi Ding ( $\nabla$ dingxuezhi@caas.cn )

Chinese Academy of Agricultural Sciences https://orcid.org/0000-0002-2113-5161

\section{Research}


Keywords: Intestinal microbiota assembly, Host-microbiome interactions, Maternal microbial transmission, Natural grazing yak

Posted Date: February 10th, 2021

DOI: https://doi.org/10.21203/rs.3.rs-170399/v1

License: (c) (1) This work is licensed under a Creative Commons Attribution 4.0 International License. Read Full License 
1 Maternal gut microbes contribute to rapid acquisition of intestinal microbiota and high-altitude adaptation of pre-weaning yak calves

Jianbo Zhang ${ }^{1,2 \dagger}$, Zeyi Liang ${ }^{1 \dagger}$, Renqing Ding $\mathrm{Kao}^{3}$, Jianlin $\mathrm{Han}^{4,5}$, Mei Du ${ }^{1}$, Anum Ali Ahmad ${ }^{1,6}$, Shengyi

Wang ${ }^{2}$, Ghasem Hosseini Salekdeh ${ }^{7}$, Ruijun Long ${ }^{6}$, Ping Yan ${ }^{1 *}$, Xuezhi Ding ${ }^{1,2 *}$

${ }^{1}$ Key Laboratory of Yak Breeding Engineering, Lanzhou Institute of Husbandry and Pharmaceutical Sciences,

Chinese Academy of Agricultural Sciences, Lanzhou 730050, China. ${ }^{2}$ Key Laboratory of Veterinary Pharmaceutical

Development, Ministry of Agricultural and Rural Affairs, Lanzhou Institute of Husbandry and Pharmaceutical

Sciences, Chinese Academy of Agricultural Sciences, Lanzhou 730050, China. ${ }^{3}$ Gannan Institute of Animal

Husbandry Science, Hezuo 747000, China. ${ }^{4}$ Livestock Genetics Program, International Livestock Research Institute

(ILRI), Nairobi 00100, Kenya. ${ }^{5}$ CAAS-ILRI Joint Laboratory on Livestock and Forage Genetic Resources, Institute

of Animal Science, Chinese Academy of Agricultural Sciences (CAAS), Beijing 100193, China. ${ }^{6}$ School of Life

Sciences, Lanzhou University, Lanzhou 730020, China. ${ }^{7}$ Department of Systems Biology, Agricultural Biotechnology

Research Institute of Iran, Agricultural Research, Education, and Extension Organization, Karaj, Iran.

Email addresses for all authors: zhangjb9122@163.com; liangzeyi1@163.com,

dingkao2020@163.com; H.JIANLIN@CGIAR.ORG; dumeicaas@126.com;

anum2017@1zu.edu.cn; wangshengyi@ caas.cn; hosseini.salekdeh@mq.edu.au;

longrj@1zu.edu.cn; pingyanlz@163.com; dingxuezhi@caas.cn

*Correspondence: pingyanlz@163.com; dingxuezhi@caas.cn

†These authors have contributed equally to this study. 


\section{Abstract}

Background: Long time exposure to seasonal forage availability and harsh environment on the Qinghai-Tibetan Plateau (QTP) has resulted in a series of unique adaptation mechanisms following the evolution of yak to cope with nutritional deficiencies and other adverse conditions. This is likely achieved by an unprecedented genetic resource for fibrolytic enzymes of microbial origins that allow the host to efficiently degrade plant polysaccharides. However, to what extent of maternal symbiotic microbial transmission throughout early microbial successions and its adaptation to highaltitude hypoxia in grazing yak driven by the harsh environment and nutritional stress have been far from clear. Understanding the colonization and succession of yak gut microbiota would help to clarify the functional interaction and crosstalk between microorganisms and their hosts. This study explored the succession of intestinal microbiota of yak (Bos grunniens) and cattle (Bos taurus) kept in the same habitat during pre-weaning period.

Results: The gut microbiota of yak and cattle calves were dominated by members of the families Ruminococcaceae, Lachnospiraceae, and Bacteroidaceae during pre-weaning. Moreover, sourcetracking models revealed that maternal microbiota was critical for the rapid establishment and colonization of initial intestinal microbiota of their calves at development stage and its impact persisted until weaning or even longer. Compared to cattle calves, the gut microbiota of yak calves was rapidly established and reached to a relatively stable status at the 5th week after birth, indicating the evolutionary significance of interaction between the yak and its intestinal microbial community that could facilitate the adaptation of this flagship species to adapt to the harsh environment on the QTP.

Conclusion: Our results revealed that under natural grazing conditions, the calves raised by their 
mothers acquire gut microbiota through the contacts with maternal feces and the social learning behavior, which accelerate the establishment of stable intestinal microbiota. In addition, after longterm natural selection, the yak calves acquire a relatively mature and stable intestinal microbiota earlier than the cattle calves, facilitating their strong adaptation to the harsh environment on the QTP.

Keywords: Intestinal microbiota assembly, Host-microbiome interactions, Maternal microbial transmission, Natural grazing yak

\section{Background}

The animal gastrointestinal tract is inhabited by a diverse microbial community, which influences a wide range of host metabolic processes, immune system, central nervous system development, and even behavior [1-3]. This complex community of microbes must be reassembled each generation. The animal gastrointestinal tract is thought to be largely sterile in newborn $[4,5]$, and is subsequently colonized by diverse bacterial taxa varying in abundance and functional traits $[2,3]$. Insight into the establishment and composition of microbial communities is essential for predicting and directing their future states[6]. There is increasing evidence that microbial colonization is a complex process influenced by a two-way interaction between the host and microbial community as well as a variety of external factors, such as neonatal delivery, maternal and environmental microbiota, diet, parenting behavior, and early use of antibiotics [7-11]. Studies have revealed the essential roles of environmental and maternal microbes in the establishment of newborn microbiota, which is likely critical for protecting the newborn from pathogens when its immune system is immature [12-14]. However, ruminants are born with underdeveloped rumen, reticulum, and omasum are considered functionally monogastric animals before weaning [15]. Therefore, dietary nutrients obtained from hindgut is likely an important source of energy for ruminants throughout all 
stages of their development, while hindgut fermentation could be of an elevated importance to the calves during their first days of life before the rumen is fully developed [16]. Some studies have shown that microbial fermentation in the hindgut may be responsible for up to $30 \%$ of cellulose and hemicellulose degradation in ruminants $[17,18]$. There are a few studies that examined the intestinal and fecal microbiota in newborn calves [19, 20]. In contrast to that of rumen microbial ecosystem, the fundamental role of intestinal microbiota and its contribution to ruminant health and production in newborn calves are less well understood. In addition, knowledge about the possible sources of early gastrointestinal microbiota and their colonization may help to explore the functional interaction between host metabolism and gut microbiota.

The yak (Bos grunniens), a herbivore exclusively inhabiting the Qinghai-Tibetan Plateau (QTP) and adjacent mountainous regions, evolutionarily diverged from cattle (Bos taurus) about 4.4 to 5.3 million years ago [21]. The QTP offers one of the harshest environments for the survival of humans and other mammalian species. It has been found that yak are superior to cattle in feeding and grazing behavior [22], digestive organ structure [23, 24], nitrogen use efficiency [25], low rumen methane emission [26], and interseason energy utilization efficiency [27, 28]. A whole genome sequencing study has identified potentially functional genes related to the unique adaptation of yak to severe hypoxia condition [29]. A recent study argued that yak adaptation mechanisms to harsh environment and long-term nutritional stress on the QTP are related to the enrichment of key genes for volatile fatty acid (VFA) fermentation pathway in rumen microbiome while methanogenesis pathway are enriched in cattle [30]. Furthermore, rumen microbial compositions change during the growth of yak from neonatal (7 days) to adult (12 years) stages, especially the bacterial and archaeal groups are more sensitive in response to development stages compared to the two eukaryotic microbial 
groups [31]. Notably, most of the above-mentioned studies focused on the structure, function, and succession of the rumen microbial community in yak, but there is limited knowledge about how intestinal microbiota develops during pre-weaning period and when it becomes fully established during yak lifetime.

Some studies have shown that maternal microbes quickly colonize offspring gut after parturition through birth canal, skin contact or breast feeding and shape the onset of an intestinal immune system and its future development [32-34]. Yak calves are mainly fed by maternal nursing under traditional natural grazing condition during the pre-weaning period, which is beneficial for the calves to learn how to eat forage earlier and to promote the development of gastrointestinal tracts. However, the weaning time of yak calves is prolonged, which greatly limits the productivity of yak [35]. We hypothesize that a core intestinal microbiota develops in yak calves and facilitates their strong adaptation to harsh environment on the QTP, and that the intestinal microbial community structure of yak calves got established and matured earlier than that of cattle calves under the same habitat. Therefore, we collected fecal samples in yak and cattle calves from one to nine weeks after birth along with rumen fluid, feces, oral cavity and breast surface swabs of their mothers at one week post parturition for $16 \mathrm{~S}$ rRNA amplicon sequencing. We aimed to track down the sources, determine the time of establishment, and explore the phylogenetic composition of the intestinal microbiota of yak calves, which are expected to shed light on the fundamental knowledge essential to the development of strategies to improve the intestinal function of yak.

\section{Methods}

\section{Animals experiments and sampling}



on the same native pasture (without concentrate supplementation) in Yangnuo Specialized Yak Breeding Cooperative $\left(34^{\circ} 43^{\prime} 19.66^{\prime \prime} \mathrm{N}, 102^{\circ} 28^{\prime} 49.51^{\prime \prime E}\right)$ at Xiahe county of Gannan Tibetan Autonomous Prefecture, Gansu Province, China. All the animals involved in this experiment were from the same herd and they all grazed together in an alpine meadow on the QTP, where the average altitude is $3,300 \mathrm{~m}$ and the average annual temperature is $4{ }^{\circ} \mathrm{C}$. There were abundant natural alpine meadow herbage and water resource, and the animals freely drank water from the local river or the snow meltwater. In addition, all the animals grazed from 7 a.m. to 6 p.m., and the samples were collected before the morning grazing. All animals included in this study were healthy during our sampling period and received no recorded therapeutic or prophylactic antibiotic treatment. birth. Rumen fluid, feces, oral cavity, and breast skin swab from their mothers were also sampled at one week post parturition. An overview of the experimental design is shown in Fig. 1. Briefly, feces pump prior to the morning grazing, while oral cavity (YOc: $\mathrm{n}=8 ; \mathrm{COc}: \mathrm{n}=8$ ) and breast surface

130 (YBs: $\mathrm{n}=8$; CBs: $\mathrm{n}=8$ ) samples were obtained by swabbing mouth and breast with a sterile cotton 
A Sampling Area

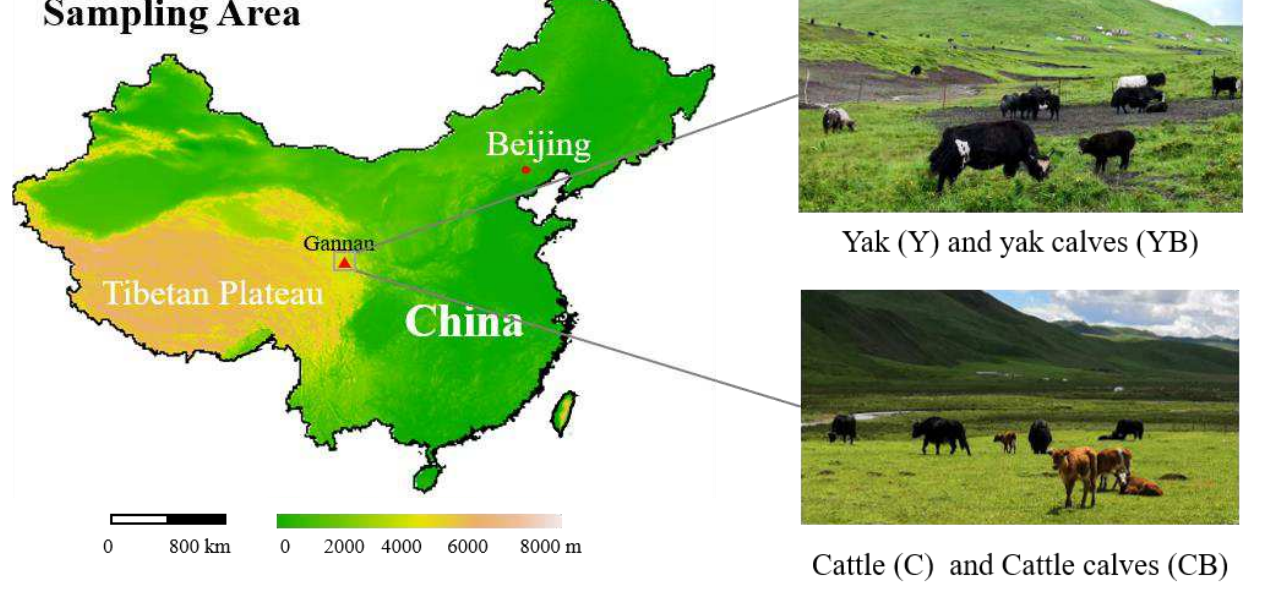

B Samples Types

Maternal Microbiota
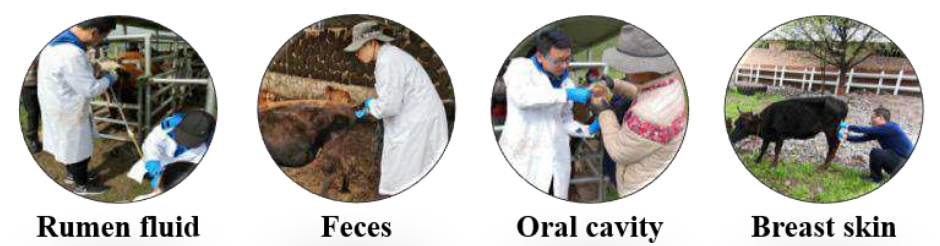

YR, $\mathrm{n}=8$

YF, $\mathrm{n}=8$

Oral cavity

Breast skin

$\mathrm{CR}, \mathrm{n}=8$

$\mathrm{CF}, \mathrm{n}=8$

YOc, $\mathrm{n}=8$

YBs, $\mathrm{n}=8$

COc, $\mathrm{n}=8$

CBs, $\mathrm{n}=8$

Pre-weaning

Calves Microbiota

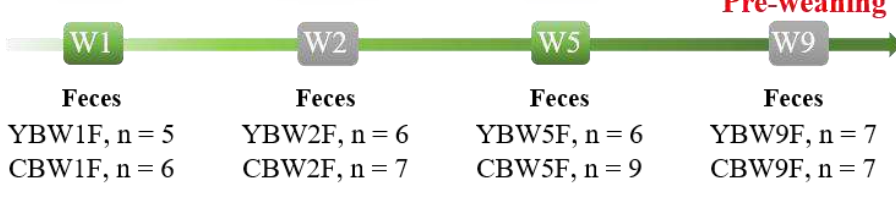

Fig. 1 Intestinal microbial source and succession analysis of yak (YB) and cattle $(\mathrm{CB})$ calves on the same pasture at

different weeks during pre-weaning.

\section{DNA extraction and Illumina sequencing of 16S rRNA genes}

The samples included the feces from calves at four-time points (yak calves: $\mathrm{n}=24$; cattle calves: 
DNA isolation procedure. Sample preparation was applied prior DNA isolation to optimize microbial loads for 16S rRNA gene PCR amplifications.

Total genome DNA from all the samples was extracted using CTAB method. DNA concentration and purity were monitored on $1 \%$ agarose gels. DNAs were diluted to a final concentration of 1 $\mathrm{ng} / \mu \mathrm{l}$ using sterile distilled water. The bacterial V4 region of 16S rRNA gene was amplified using F515/R806 primers under the following conditions: initial denaturation at $98^{\circ} \mathrm{C}$ for $1 \mathrm{~min}$, followed by 30 cycles of denaturation at $98{ }^{\circ} \mathrm{C}$ for $10 \mathrm{~s}$, annealing at $50{ }^{\circ} \mathrm{C}$ for $30 \mathrm{~s}$, and elongation at $72{ }^{\circ} \mathrm{C}$ for $30 \mathrm{~s}$, and finished by a final extension at $72{ }^{\circ} \mathrm{C}$ for $5 \mathrm{~min}$. Amplicons were purified with Qiagen Gel Extraction Kit (Qiagen, Germany). Sequencing libraries were generated using TruSeq® DNA PCR-Free Sample Preparation Kit (Illumina, USA) following manufacturer's recommendations and index codes were added. The library quality was assessed on the Qubit@ 2.0 Fluorometer (Thermo Scientific) and Agilent Bioanalyzer 2100 system. At last, the library was sequenced on an Illumina NovaSeq platform and 250 bp paired-end reads were generated (Novogene, Tianjin, China).

\section{Bioinformatic and statistical analysis}

Paired-end reads were assigned to samples based on their unique barcode and then merged using FLASH (Version 1.2.7, http://ccb.jhu.edu/software/FLASH/) [36]. Quality filtering of the raw tags was performed under specific filtering conditions to obtain the high-quality clean tags [37] according to QIIME (Version 1.9.1, http://qiime.org/scripts/split_libraries_fastq.html) [38]. Sequences with $\geq 97 \%$ similarity were assigned to the same OTUs by Uparse software (Version 7.0.1001, http://drive5.com/uparse/) [39]. Representative sequence for each OTU was screened for further annotation. For each representative sequence, the Silva Database (http:/www.arb-silva.de/) [40] was used based on Mothur (Version 1.36.0) algorithm to annotate taxonomic information. 
163

Alpha diversity was applied to analyze the complexity of species diversity through Chao 1 species richness and Shannon diversity index using QIIME and displayed with R software (Version 2.15.3). For beta-diversity, beta_diversity.py in QIIME was used to obtain distance matrices and Principal Coordinate Analysis (PCoA) was performed and visualized using ggplot2 package in R software. Nonmetric multidimensional scaling (NMDS) plots of the Bray-Curtis metric were calculated with square root transformed data and visualized in $\mathrm{R}$ (vegan package). Permutational multivariate analysis of variance (PERMANOVA) was used to examine the differences of gut microbial communities between calves and their mothers among different age groups. The linear discriminant analysis (LDA) effect size (LEfSe) algorithm was used for differential analysis to identify significantly different taxa [41]. In addition, we used SourceTracker2 [42], a Bayesian communitylevel microbial source-tracking tool, to estimate the proportion of sequences in the calf gut microbiota that originated from different parts of their mother's body. SourceTracker2 was run with default parameters using nonrarefied data; each calf gut microbial community was designated as a sink, and all maternal sample types were designated as sources. In order to identify and sort the genera which contributed the most to the differences in gut microbial community between yak and cattle calves at different developmental stages before weaning, a similarity percentage analysis (SIMPER) was performed using PAST (Version 3.1.7) [43]. All genera below a certain threshold $(90 \%)$ of cumulative contributions were set as specialized genera. In this analysis, SIMPER determined the specialized genera in the calf gut microbiota based on the changes in cumulative contributions to explain differences among developmental stages and species groups. Moreover, intersections between sets of OTUs were visualized using UpSet plot (with the R package UpSetR (Version 1.3.3) [44]. The 16S function prediction was employed to standardize the OTU abundance 
by PICRUSt [45], which was used to remove the effect of the number of copies of the $16 \mathrm{~S}$ marker gene in the species genome. The predicted functional contents were summarized at KEGG pathway hierarchy levels 2 and 3 for interpretation and subsequent analysis.

\section{Data availability}

All raw high-throughput sequencing data were submitted to the National Center for Biotechnology Information (NCBI) Sequence Read Archive (SRA) under the Bioproject accession number PRJNA688363.

\section{Results}

\section{Richness and diversity of calves and maternal microbiota}

To assess the dynamic microbiota variations, fecal samples of yak $(n=24)$ and cattle $(n=29)$ calves were collected from the 1st to the 9th week after birth (Fig. 1). In order to explore possible sources of these microbiota, rumen fluid, feces, oral cavity, and breast skin samples were collected from their mothers $(n=8)$ in the first week after their parturition (Fig. 1). All DNA samples were initially subjected to ribosomal marker gene amplification and sequencing, and 10,029,364 qualityfiltered 16S rRNA gene sequences were obtained with an average of $85,721 \pm 9,173$ (mean \pm SD) reads per sample. The coverage ranges of different sample types were narrower (feces: 66,720 99,894; rumen: 80,673 - 99,593; oral cavity: 64,823 - 98,614; and breast skin:80,295 - 96,729; see species diversity in Additional file 1: Figure S1) than that of the total range $(64,823-99,894)$. Besides, through annotation of the sequences of the OTUs with the Silva132 database following the $97 \%$ sequence similarity threshold, a total of 13,807 OTUs were annotated, of which the proportion of sequences annotated at the genus levels were 4,463 OTUs (31.09\%). 
We found that the calves and maternal microbiota varied across different sample types. The maternal samples (rumen fluid, feces, breast skin, and oral cavity) harbored higher OTU richness than samples from the gut of calves during pre-weaning periods in both yak and cattle (Fig. 2). To gain insight into the diversity of fecal microbial community, we compared the Chaol species richness and Shannon diversity indexes of yak and cattle calves between different age groups (Fig. 2A, B, and Additional file 1: Table S1). In the intestinal microbiota of two-week old yak and cattle calves, Chao1 richness was not significantly different between the two species (YBW1F vs. CBW1F, Wilcox test, $P=0.995$; YBW2F vs. CBW2F, Wilcox test, $P=0.335$ ). Interestingly, at five weeks of age, the Chao1 species richness and Shannon diversity indexes of fecal microbiota in yak calves suddenly decreased, which was significantly different from that of cattle calves (Chao1, YBW5F vs. CBW5F, Wilcox test, $P=0.014$; Shannon, YBW5F vs. CBW5F, Wilcox test, $P=0.008$ ). At the 9th week, the differences in Chao1 species richness and Shannon diversity indexes between yak and cattle calf fecal microbiota were not statistically significant (Chao1, YBW9F vs. CBW9F, Wilcox test, $P=0.855$; Shannon, YBW9F vs. CBW9F, Wilcox test, $P=0.070$ ), indicating that the calf intestinal microbiota were relatively matured and got stabilized during this period (Additional file 1: Table S1). However, we found that although the difference in gut microbial abundance and diversity between yak and cattle were not significant with age (Chaol, YF vs. CF, Wilcox test, $P=$ 0.502; Shannon, YF vs. CF, Wilcox test, $P=0.433$ ), there were still significant differences in gut microbial abundance and diversity between calves and their mothers before weaning (Chao1, Wilcox test, YBW9F vs. YF: $P<0.001$, CBW9F vs. CF: $P<0.001$; Shannon, Wilcox test, YBW9F vs. YF: $P=0.022$, CBW9F vs. CF: $P=0.238$ ), indicating that the microbial community in the 
S1). Besides, we also found that the microbial richness and diversity of the rumen, oral cavity, and breast skin of yak cows were higher than those of cattle cows, but insignificant (Wilcox test, $P>$ 0.05) (Fig. 2A, B and Additional file 1: Table S1).

We next sought to examine how the maternal microbial community influenced the gut microbial community of both yak and cattle calves kept in the same habitat during pre-weaning (Fig. 2C, D). Principal coordinate analysis (PCoA) was performed on maternal microbiota (fecal, rumen fluid, oral cavity, and breast skin) and calf fecal microbiota at 1,2, 5, and 9 weeks of life using unweighted and weighted UniFrac distances matrices. The PCoA plot showed clear separations between the types of calf fecal and maternal samples (rumen fluid, oral cavity, and breast skin) in both species, for examples, maternal oral cavity and breast skin samples clustered together, all maternal and calf fecal samples formed a separate cluster, while maternal rumen samples stood alone (Fig. 2C, D). In addition, analysis of molecular variance (AMOVA) [46] confirmed these stratifications among the sample types (Additional file 1: Table S2). For calf fecal samples, there was no significant difference between the yak and cattle calf groups at one and two weeks after birth (AMOVA, $P>0.05$; Additional file 1: Table S2). However, the fecal microbial communities between the yak and cattle calves were significantly different at their five weeks of age (AMOVA, $P=0.043$ ). Nevertheless, such differences disappeared at their nine weeks of age (AMOVA, $P>0.05$; Additional file 1: Table S2).

Of the 29 bacterial phyla identified, six dominated the calf fecal microbiota (average cumulative abundance $=98.5 \%$, including Firmicutes, Bacteroidetes, and Proteobacteria being prevalent in adult bovine intestinal microbiota [47] (Fig. 2E). Firmicutes (YB: 55.9\%; CB: 62.7\%) were the most dominant phylum among both the calf and maternal gut microbiota (rumen and hindgut), followed 
by Bacteroides (YB: 35.1\%; CB: 30.5\%). However, Proteobacteria was the most dominant phylum in oral cavity (YOc: $61.2 \%$, COc: $76.3 \%$ ) and breast skin microbial communities (YBs: $36.5 \%$; CBs:35.5\%) of maternal origins, followed by Firmicutes. It is worth noting that Actinobacteria was also common in the maternal breast skin microbiota (YBs: 16.3\%; CBs: 13.0\%) (Fig. 2E). Interestingly, Euryachaeota (YB: 0.016\%; CB: 0.003\%) and Thaumarchaeota (YB: 0.004\%) were found in the feces of both yak and cattle calves at their 1st week of age, indicating that archaea had colonized the calf intestines very early. Lactobacillales (YB: 5.9\%; CB: 4.8\%) were the three most abundant orders in the intestines of yak and cattle (Additional file 1: Figure S2). At the family level, we found that Ruminococcaceae (YB: 24.8\%; CB: 30.5\%), Lachnospiraceae (YB: 13.5\%; CB: 17.8\%), and Bacteroidaceae (YB: 15.1\%; CB: $11.1 \%$ ) were prevalent in the gut of both pre-weaning yak and cattle calves (Fig. 2F). Moreover, Lactobacillus was abundant in the gut microflora of both yak and cattle calves at their very early age, but its abundance decreased gradually at their ages in later weeks. In comparison with the intestinal microbiota, the oral cavity and breast skin microbiota of both yak and cattle cows mainly consisted of Moraxellaceae (YOc: 15.2\%, COc: 33.6\%; YBs: 14.6\%; CBs: 18.1\%) followed by communities in the gut of both yak and cattle calves increased along with their ages (Fig. 2F). 


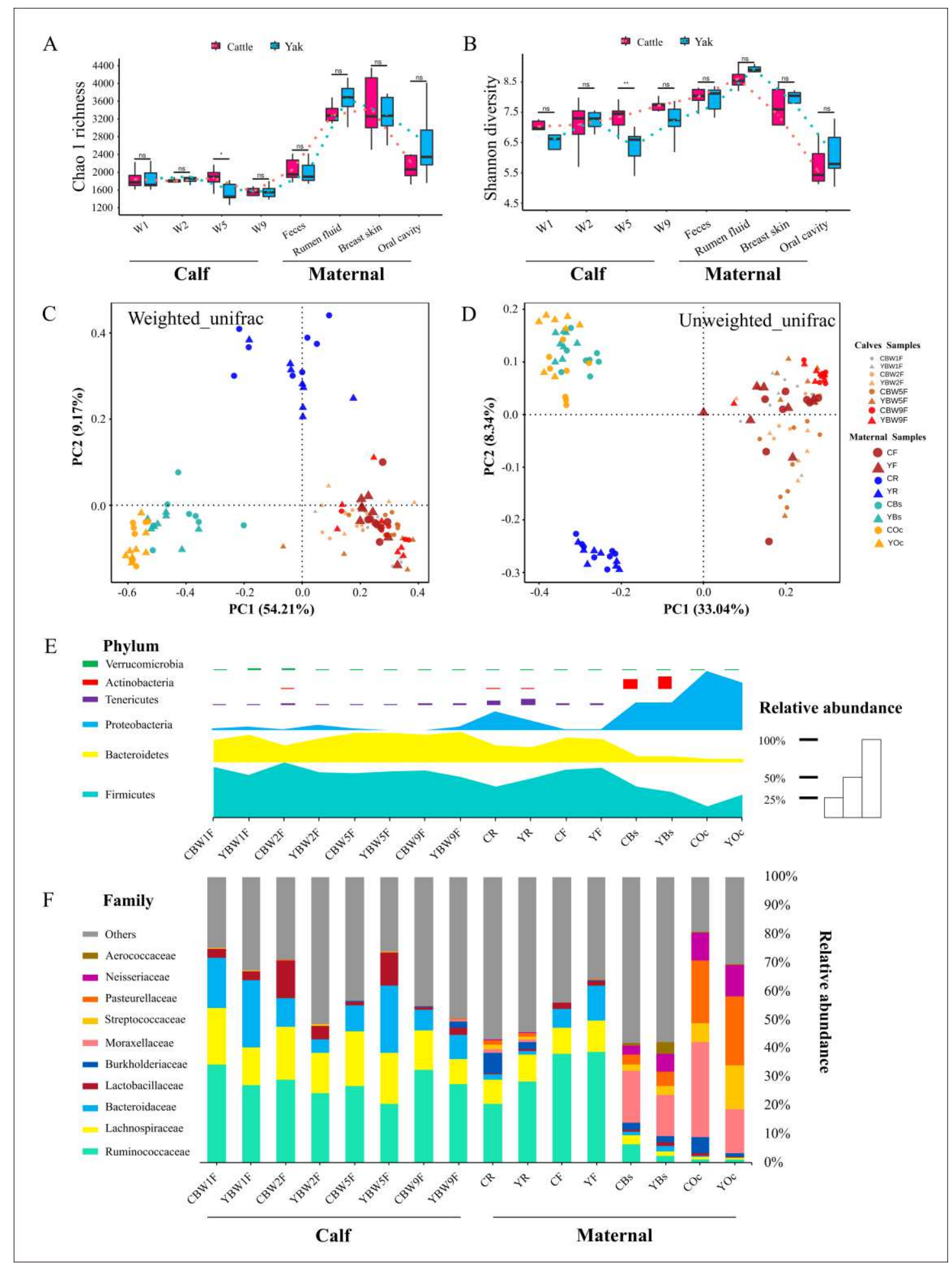

Fig. 2 Compositions of the calf and maternal microbiota. (A) The Chaol species richness and (B) Shannon diversity

271 indexes of microbial communities in the samples of yak and cattle calf feces (at 1, 2, 5, and 9 weeks of their ages),

and their mothers (rumen fluid, feces, oral cavity, and breast skin). Distances between the samples, based on OUT

273 similarity (OTU similarity $\geq 97 \%$ ) calculated using (C) weighted UniFrac distances in all samples; and (D) 
unweighted UniFrac distances in all samples; both visualized in PCoA plots. A greater distance between two points infers a lower similarity, whereas similar OTUs cluster together. (E) The average relative abundances of the most prevalent bacterial phyla (bar length and area plot) in each sample type are plotted for samples from the maternal microbiota and calf feces. (F) Bar plots depict the relative abundances of bacterial families from the maternal samples and calf feces; unclassified taxa and bacterial families which have a relative abundance less than $1 \%$ were grouped into "Others"

\section{Predictive source tracking of the calf gut communities during pre-weaning}

Bayesian community-level source tracking [48] was used to investigate the contribution of maternal microbiota to the gut microbial community assemblies of both yak and cattle calves. The SourceTracker model also revealed the main roles of the microbial communities from different body parts (rumen fluid, feces, oral cavity, and breast skin) of both yak and cattle cows in shaping the intestinal microbial communities of their calves. It was clear that the intestinal microbiota of both yak and cattle calves at their early ages were primarily derived from the maternal intestinal microbiota (Fig. 3). On average, $94.1 \%$ of the intestinal microbiota of yak calves at 1st week of age were from maternal intestinal source while less than $0.2 \%$ from maternal rumen and $5.7 \%$ from unknown sources (Fig. 3A); Comparatively, 94.3\% of intestinal microbiota of cattle calves also originated from maternal intestinal source, but less than $0.3 \%$ from maternal rumen and $5.4 \%$ from unknown sources (Fig. 3B). On average, both yak and cattle calves at 2nd week of their age still carried more than $80 \%$ of such maternally-originated gut microbial community. However, the proportions from the maternal rumen fluid, breast skin, and other unknown sources started to increase along with their ages. Similarly, UpSet analysis revealed that the maternal microbial community played an important role in the succession of the intestinal microbial community of both 
yak and cattle calves after birth, especially the maternal intestinal microbial community (Fig. 3).

The result was consistent with the patterns derived from the taxonomy and linear analyses (Fig. 2).

298 Moreover, in order to further determine the influence of the maternal gut microbial community on

299 the succession process of the calf's early gut microbiota, NMDS analysis was performed to

300 determine whether the microbial community structure changed along with their ages. The similarity cluster analysis based on NMDS showed a good agreement with the result of source tracking (Fig. 4F), that is, the gut microbial communities of both yak and cattle calves at the 1st and 2nd weeks of their ages were more similar to those in the guts of their mothers. Hence, both SourceTracker and UpSet analyses indicated that their mothers cares contributed to the rapid establishment of intestinal microbial community of the calves, and especially that the maternal intestinal microbiota significantly shaped the composition and stability of the calf intestinal microbial community.

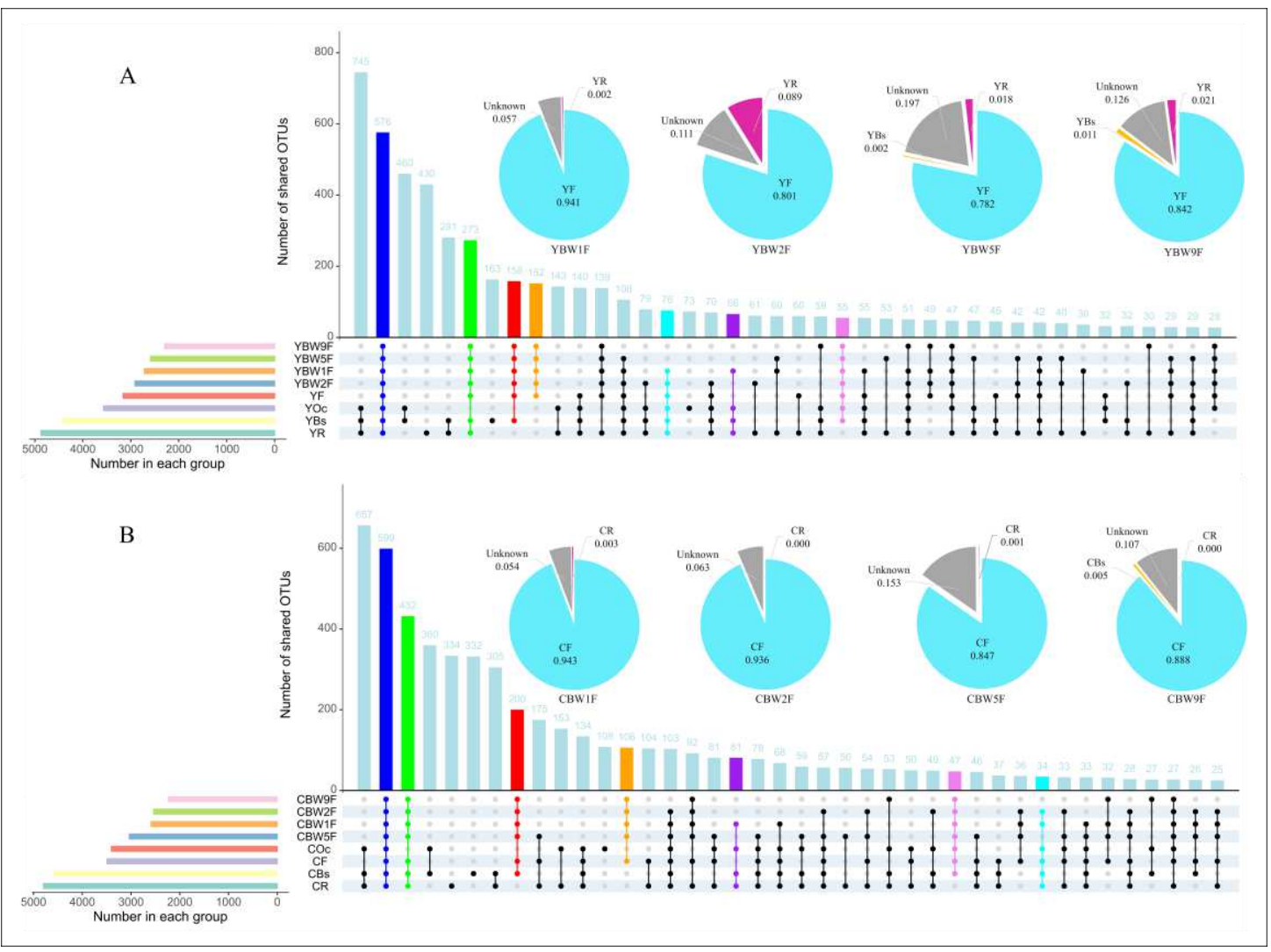

Fig. 3 Community-level modeling and source-tracking analyses of the maternal sources in early calf gut community 
assembly. Yak (A) and cattle (B) animals are displayed separately. UpSet plots of common OTUs in the samples from the calf feces (at 1, 2, 5, and 9 weeks of their ages) and their mothers (rumen fluid, feces, oral cavity, and breast skin). Only OTUs with an overall abundance across all samples greater than $0.01 \%$ are included, the 40 intersections which involved the greatest number of OTUs are displayed. The pie charts show the predicted proportions of sequences in the gut microbiota of both yak and cattle calves at different weeks pre-weaning $(1,2,5$ and 9 weeks) that originated from their maternal microbial communities (rumen fluid, feces, oral cavity, and breast skin).

\title{
Influence of the maternal gut microbiota on early calf gut microbial succession
}

\author{
To determine the association between early gut microbial succession and mother care, the
} resemblance between gut microbiota of calves and their mothers was detected by performing LEfSe in given communities (Fig. 4 and Additional file 1: Figure S3). The gut microbiota of yak cows were enriched in Actinomycetaceae, Methylopilaceae, and Chthoniobacteraceae, while cattle cows were enriched in Kineosporiaceae, Barnesiellaceae, and Rikenellaceae at the family level (Fig. 4E). At genus level, the gut microbiota of yak cows were enriched in Kandleria, Methanosphaera, and Cupriavidus, while cattle cows were enriched in Butyrivibro, Acetitomaculum, and Acinetobacter (Additional file 1: Figure S3E). Interestingly, Pyrinomonadaceae, Actinomycetaceae, Bifidobacteriaceae, and Methanobacteriaceae were the dominant families of gut microbes in yak calves at the 1st week of their ages (Fig. 4A and Additional file 1: Figure S3A). However, the relative abundances of Proteobacteria, Fibrobacteria, Cyanobacteria, and Spirochaetes in the intestinal microflora of yak calves at the 2nd week was higher than those of cattle calves, but Elusimicrobia, Planctomycetes, and Kiritimatiellaeota (at phylum level, Fig. 4C) in the intestinal microflora of cattle calves at the 5th weeks were higher than those of yak calves. In addition, the LEfSe result showed that the gut microbiota of yak calves at the 2 nd week were enriched for 

the genera level, Fig. 4B and Additional file 1: Figure S3B). Following the increase in age, the differences in the microbial communities in the intestines between yak and cattle calves decreased gradually. We found that the gut microbiota of yak calves at the 9th week were enriched in Chitinophagaceae, Peptostreptococcaceae, and Fusobacteriaceae, while cattle calves were enriched in Christensenellaceae, Lachnospiraceae, and Peptococcaceae (at the family level, Fig. 4D). Therefore, the LEfSe result further demonstrated that maternal gut microbiota was the major contributor to calf initial gut community. of the calf early gut microbiota, NMDS analysis was performed to determine whether the microbial In order to further determine how the maternal gut microbiota influence the succession process community structure changed with increasing age (Fig. 4F). The similarity cluster analysis based on NMDS (stress $=0.181$ ) showed a good agreement with the result of LEfSe analysis (Fig. 4F and Additional file 1: Table S4). In one-week old calves, the intestinal microbial communities were similar between yak and cattle (YBW1F vs. CBW1F, PERMANOVA, $\mathrm{F}_{1,9}=0.868, \mathrm{R}^{2}=0.088, P$ $=0.514 ;$ Additional file 1: Table S4). Along with the gradual development of intestinal tract, the Bata diversity in the intestinal microbial communities of calves at different weeks of age (2 and 5 weeks after birth) and from different species (yak and cattle) was significantly different (YBW2F vs. CBW2F, PERMANOVA, $\mathrm{F}_{1,11}=2.084, \mathrm{R}^{2}=0.159, P=0.012$; YBW5F vs. CBW5F, PERMANOVA, $\left.\mathrm{F}_{1,13}=2.184, \mathrm{R}^{2}=0.144, P=0.014\right)$. Finally, the intestinal microbiota of yak and cattle calves gradually got matured and stabilized by the time of weaning (YBW9F vs. CBW9F, PERMANOVA, $\mathrm{F}_{1,12}=1.702, \mathrm{R}^{2}=0.124, P=0.054$ ), and the gut microbiota of yak and cattle 


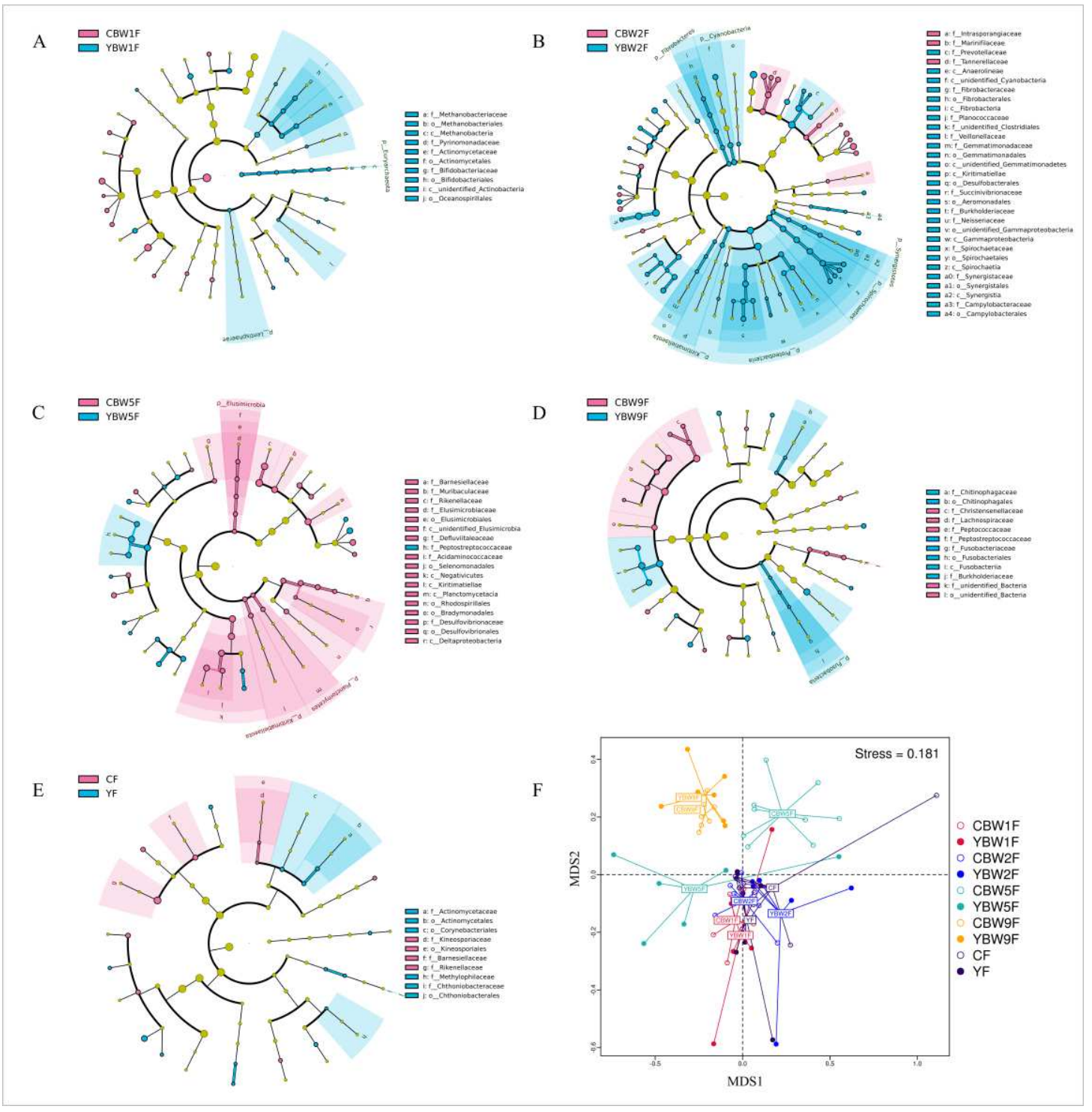

Fig. 4 LEfSe analysis. The cladograms indicate the phylogenetic distribution of the intestinal microbiota of yak and cattle at (A) 1, (B) 2, (C) 5, and (D) 9 weeks before weaning and their mothers (E) using the Linear Discriminant Analysis (LDA) Effect Size (LEfSe) method. Differences are represented by treatment colors (nattier blue indicates yak, pink indicates cattle, and yellow non-significant). Circle's diameters are proportional to the taxon's abundances. Circles represent taxonomic ranks from domain to species from inside to out layers. The LDA cut-off score is 2. 
community structures across calf and maternal samples. Non-metric multidimensional scaling (NMDS) ordination based on Bray-Curtis distances among sample types are plotted based on OTU abundances in the calf and maternal samples.

\section{Establishment of the specialized microbial communities in the guts of yak and}

\section{cattle calves pre-weaning}

The specialized community was defined based on the SIMPER analysis, to identify predominant gut microbial genera contributing to the specialized community of the intestinal microbiota of yak and cattle calves at different weeks pre-weaning. We found that only $6.8 \%$ of the overall community of fecal samples, i.e., 30 (of 443) genera with the highest variabilities in their relative abundances were responsible for $90 \%$ of the dissimilarities between the communities at different age groups of yak and cattle calves (Fig. 5). In general, the metabolic capacity of these 30 genera belonged to the specialized communities, which reflected the major differences in the intestinal microbial communities of yak and cattle calves at different weeks pre-weaning. However, there were some unique microbial communities in the intestines of yak and cattle calves at different weeks of early development. In one-week old calves, the relative abundances of Lachnoclostridium, Akkermansia and Butyricccus in the intestines of yak were higher than those of cattle, while the relative abundance of Faecalibacterium in the intestines of cattle was higher than those of yak (Additional file 1: Table S5). After 2 weeks, the relative abundances of some unidentified genera in the intestines of yak calves were higher than those of cattle calves, such as unidentified_Enterobacteriaceae, unidentified_Prevotellaceae, and unidentified_Clostridiales; however, there were significant differences in the relative abundances of certain members of Ruminococcaceae and Lachnospiraceae in the intestines between yak and cattle calves (Fig. 5 and Additional file 1: Table 
Additional file 1: Table S5).

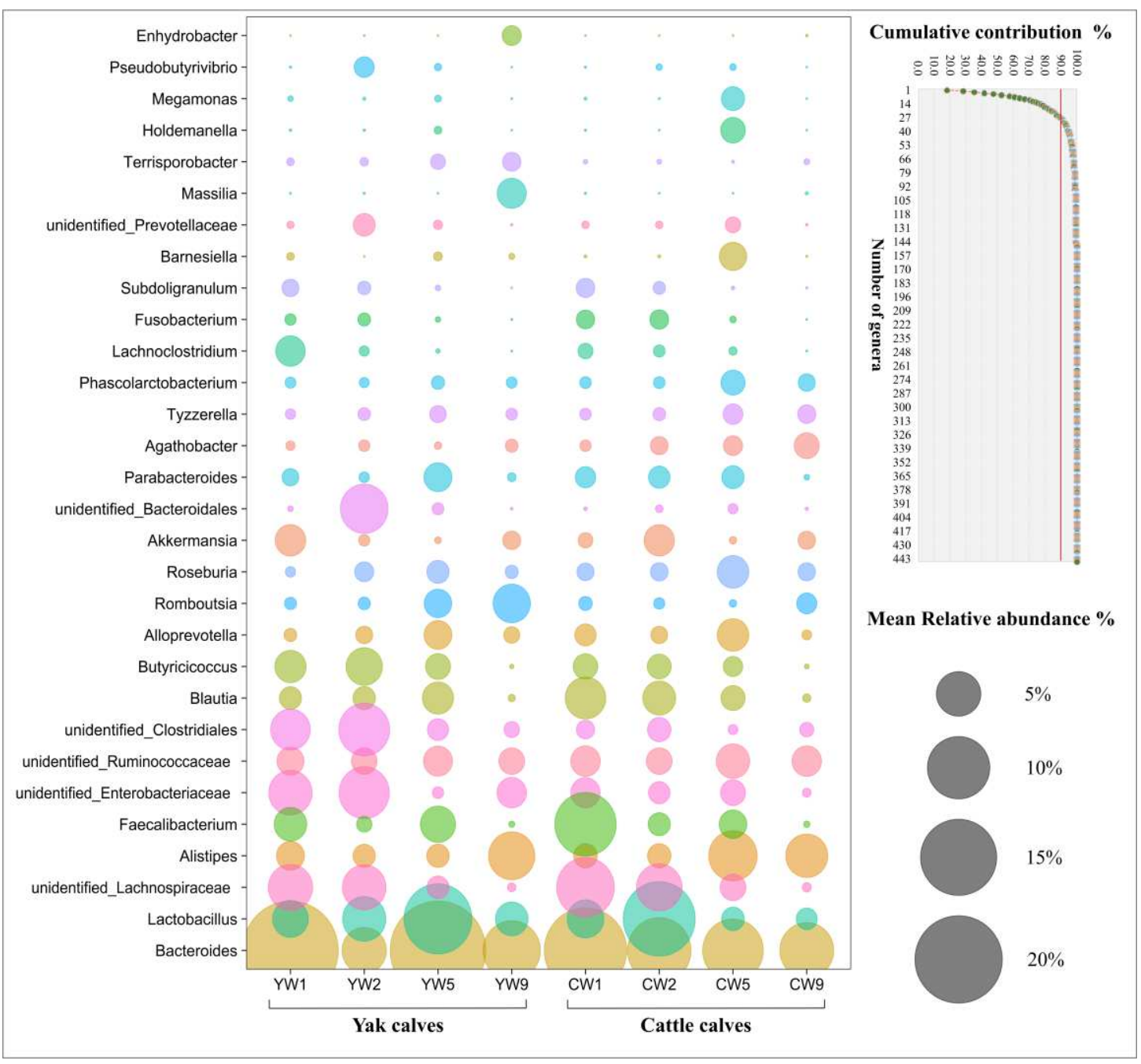

Fig. 5 Similarity percentage analysis (SIMPER). The line graph shows the result of the SIMPER analysis performed 


\section{Predicted function of the gut microbiota in pre-weaning calves}

To assess the metabolic potentials of the gut microbiome, PICRUSt-based functional prediction revealed the differences of microbial functions in the intestinal microbial communities between yak and cattle calves at different weeks pre-weaning. Generally, these differences were mainly manifested in carbohydrate metabolism, amino acid metabolism, energy metabolism, metabolism of cofactor and vitamin, and other secondary metabolites biosynthesis (Fig. 6 A). There were significant differences in the function of intestinal microbiota between yak and cattle calves in the aspects of pyruvate metabolism (T-test, $P=0.047$ ), lysine biosynthesis (T-test, $P=0.048$ ), phenylamine metabolism (T-test, $P=0.046$ ), and thiamine metabolism (T-test, $P=0.032$ ), indicating that the intestinal microbial function in yak and cattle calves within the 1st week mainly focused on carbohydrate and amino acid metabolisms (Fig. 6 B). However, at the 2nd week after birth, the intestinal microbial function of yak calves was higher than that of cattle calves in energy metabolism (T-test, $P=0.047$ ), nicotinate and nicotinamide metabolism (T-test, $P=0.032$ ), and beta-alanine metabolism (T-test, $P=0.049$ ), showing that the intestinal microbiota of yak calves had been relatively stable in nutrient digestion and metabolism (Fig. 6 B). Notably, it was predicted that the intestinal microbiota function of cattle calves at five-week of their ages was significantly higher in methane metabolism (T-test, $P=0.003$ ) than that in yak calves, which illustrated that the capability of methanogenesis in the hindgut of cattle might be higher than yak. The intestinal microbial function of yak calves at nine-week of their ages was significantly higher in carbohydrate metabolism, including butanoate metabolism (T-test, $P=0.029)$ and citrate cycle (TCA cycle) $(\mathrm{T}-$ 


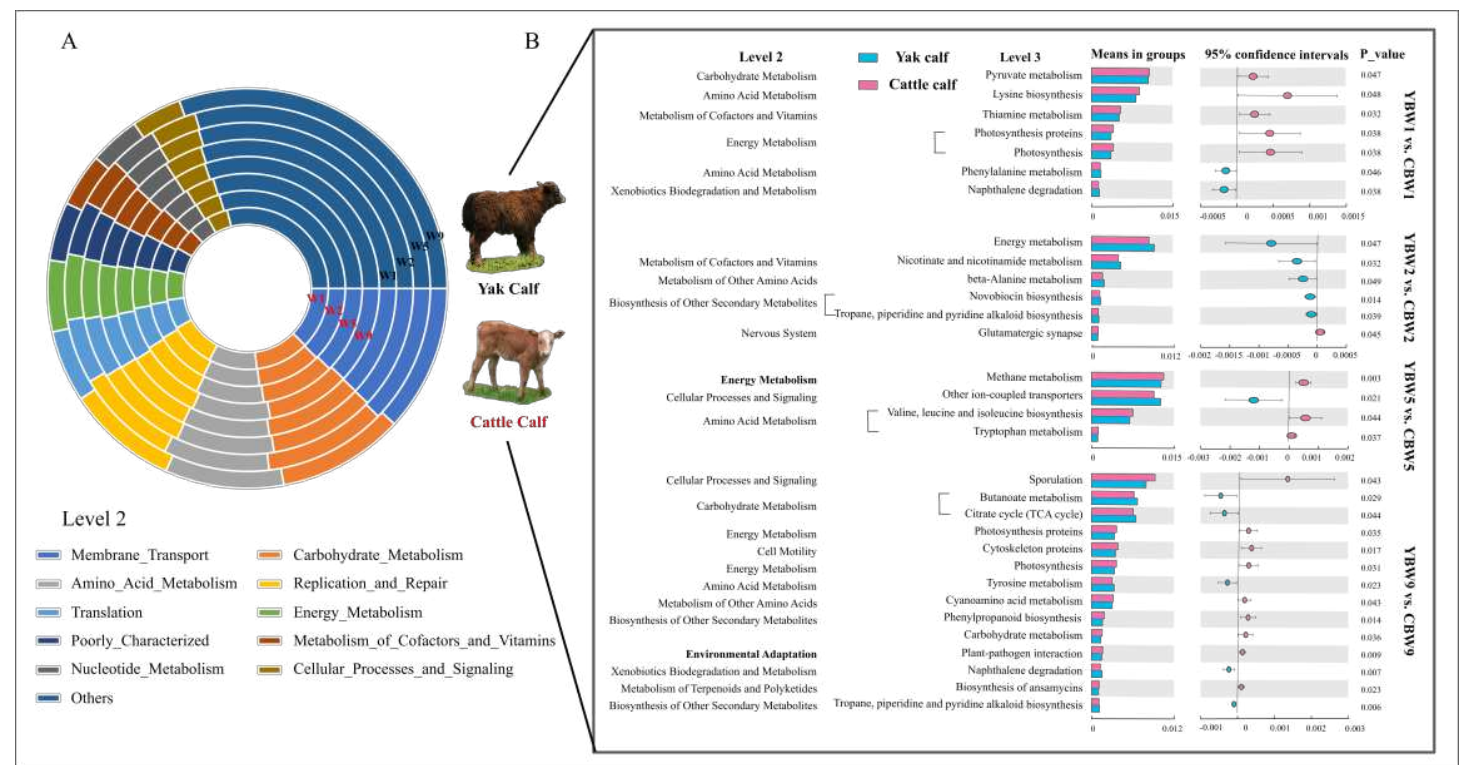

Fig. 6 Predicted microbial functions using PICRUSt. (A) Relative abundances of level 2 KEGG pathways are

\section{Discussion}

Central to ruminant production and health is the intestinal microbiota, the complex microbial 
fermentation profiles in neonatal ruminants. Hence, the objective of our study was to explore the composition and possible sources of the intestinal bacterial community in yak and cattle calves from birth until pre-weaning and to identify changes in the intestinal microbiota during normal development. In contrast to other studies that mainly examined Holstein-Friesian calves[11, 52], we analyzed gut microbiota development of yak calves, the predominant dairy and meat animals in the QTP region. Our results demonstrated that the maternal microbiome is critical for the rapid establishment of intestinal microbiota of the calves at the early development stage and has the greatest impact on the colonization of the calf intestinal microbiota within the first week after birth, and that this effect persists until weaning or even longer. This phenomenon was similar in both yak and cattle calves despite they had their respective mothers to take care of them. Besides, our results revealed that the gut microbiota of yak calves was rapidly established and relatively stable at the 5 th week compared to cattle calves. This finding indicates that yak calves have evolved to quickly establish a relatively mature and stable intestinal microbial community to adapt to harsh environment on the QTP.

\section{Maternal effects on the shaping and assembly of calf gut microbiota during early}

\section{development}

There is increasing evidence that microbial colonization is a complex process influenced by a mutual interaction between host and microbes along with a variety of external factors, such as neonatal delivery, maternal and environment microbiota, diet, parenting behavior, and early use of antibiotics [8-11, 53]. However, calves of both yak and cattle in this study were born naturally, fed by their mothers, and grazed along with their mothers on the same natural alpine pastures (without concentrate supplementation) on the QTP. Bayesian community-level source tracking and UpSet 
analyses showed that maternal fecal microbiome was critical for the rapid establishment and colonization of the intestinal microbiota in the calves within the first week of birth (94\% via maternal fecal transmission), and this effect persisted until weaning or even longer. Several studies on other species suggested that the gut microbiota was transferred from mother to offspring through social interaction, shared environment, and $\operatorname{diet}[9,10,54,55]$. In parallel, initially colonized microbiota in neonatal gut originated from the maternal vagina, breast milk and fecal microbes[5658]. Later on, a great degree of parental care may increase a variety of parental microbes in newborns during their early stage of intestinal microbiota development, such as skin microbes[59]. This process is essential for the recruitment and establishment of microbiota and helps to resist pathogens when the immune system is not well developed in newborns [12-14]. Recently, it has been reported that kid goats reared with their dam had a greater rumen development than their twins that were isolated from adult animals and fed on milk replacer, although both groups had access to the same forage and concentrate offered ad libitum [60]. Additionally, some studies found that calves reared in the presence of older companions exhibited more frequent and longer visits to the feeder, which was assumed hypothesized to be a result of social learning [61, 62]. Hence, the advantage of direct inoculation of microbiota via physical contact with the dam deserves further attention.

In contrast to most of these studies, our study focused on the influence of maternal microbiome on the calf gut microbial community during postnatal and pre-weaning development. Our results showed that since calves live with their dams and herds, the maternal intestinal microflora can be transmitted indirectly to the calves' intestines during their social feeding behavior, and it also promotes the rapid establishment and stability of calf intestinal microbiota. This phenomenon was similar in both yak and cattle calves, although they have their mothers to take care of them, further 
indicating that our results are stable and reliable. It has recently been reported that the establishment of intestinal microbiota within the first seven weeks of life is associated with calf health and growth (neonatal diarrhea, pneumonia and weight gain), while colonization by enteric pathogens might be responsible for the observed dysbiosis in gut microbiota during neonatal diarrhea [63]. Moreover, studies have found that calves are more likely to learn to eat forage in advance when they are raised with other cattle of different ages [64]. Along with this, around $22 \%$ of the change in the first milk yield of a cow is associated with the average daily gain in the first a few weeks of life [65]. Surprisingly, studies have pointed out that neonatal diarrhea is the main cause of calf death in preweaning and accounting for more than $50 \%$ of the calf deaths in dairy industry [66]. However, under the commercial dairy system, calves are usually separated from the dam at a young age and fed either milk replacer or whole milk, which increase the risk of diarrhea in calves. Therefore, we recommended that calves should be raised by their mothers during the first a few weeks, which would help to increase milk production at later stages in commercial dairy system.

In recent years, fecal microbiota transplantation (FMT) is one of the hot research directions, which is considered to be helpful to treat intestinal diseases and establish a healthy intestinal microbiota [67-69]. A proof-of-concept safety study showed that oral-fecal transplantation could shift the microbiota composition of infants who were born via cesarean section to a profile that was more similar to those born via vaginal delivery [70]. As co-author of this research, Sture Andersson, puts it, "it's a gift from mother to baby". Hence, it is suggested that the calf should live with its mother for at least one week after birth in future ruminant farming, which will help to establish and stabilize the intestinal microbiota of the calf, as well as to lower down the economic loss caused by diarrhea of pre-weaning calves. 


\section{The rapid establishment and stability of gut microbiota is the key for yak calves}

\section{to adapt to the QTP}

Studies of genetic adaptation, a central focus of evolutionary biology, most often focus on the host's genome but rarely on the co-evolved microbiota. It is well known that QTP offers one of the most extreme environments for the survival of humans and other mammalian species [21]. Yak has developed many anatomical and physiological traits to adapt to this extreme living habitat $[23,24]$.

Previous studies on rumen microbial diversity in yak found that the yak rumen microbiota has stronger efficient fiber-degrading and energy-harvesting abilities than that of cattle from low altitude [30]. However, there is a paucity of knowledge on how the intestinal microbiota develops during pre-weaning period and at what stage of life it becomes fully matured in grazing yak. Our result revealed that, except for the 5th week, there was no significant difference in the gut microbial richness and diversity between yak and cattle calves. We did not find any significant difference in the intestinal microbiota of yak calves between the 5th and the 9th week neither. This finding suggested that yak calves could quickly establish a relatively mature and stable intestinal microbial community, which may facilitate the adaptation of yak to the extreme natural environment of QTP. In addition, when microbiota composition throughout the gastrointestinal tract was explored, the rumen and intestinal regions consisted primarily of Firmicutes and Bacteroidetes, while $>55 \%$ of the bacteria in the intestine was composed of Firmicutes [47, 71]. In the present study, Ruminococcaceae, Lachnospiraceae, Bacteroidaceae, and Lactobacillaceae were the most abundant family in the gut of pre-weaning yak and cattle calves. Ruminococcaceae and Lachnospiraceae belong to butyrate-producing bacteria, indicating that they provide energy to the host by promoting the degradation of plants fibers $[72,73]$. Moreover, members of the genus 
Lactobacillus are known as probiotics to secrete lactic acid as the major end product of carbohydrate metabolism, therefore playing an important role in nutrition, growth, and protection from infection [74-76].

There is increasing evidence that microbial colonization is a complex process influenced by a mutualistic interaction between the host (immune defense mechanisms, food retention time in the gut) and microbial factors (adhesion, mechanisms to obtain nutrients from the host, and survival mechanisms under oxygen gradient). Along with this, a variety of external factors, such as neonatal delivery, maternal and environment microbiota, parenting behavior, and early use of antibiotics, all combine to influence gut colonization $[8-11,53]$. To determine the association between early gut microbial successions and mother care, LEfSe analysis was performed to identify representative taxa in given communities. Interestingly, we found that Pyrinomonadaceae, Actinomycetaceae, Bifidobacteriaceae, and Methanobacteriaceae were the dominant families of gut microbiota in yak calves at 1 week of life. The study showed that the original colonizers (Streptococcus and Enterococcus) utilize available oxygen in the intestinal and create an anaerobic environment for the strict anaerobic intestinal residents (such as Bifidobacteria and Bacteroides) [77, 78]. Besides, our study has shown that the administration of Bifidobacterium and Lactobacillus to newborn calves during the first week of life increased weight gain and feed conversion ratio, while decreased diarrhea incidences [79]. Our finding indicated that the relative abundance of some microbial communities such as Prevotellaceae, Acidaminococcus, Fibrobacter, Ruminobacter, Succinivibrio, Lachnospira, etc., in the intestines of yak calves were significantly higher than cattle calves, although there was no significant difference in Chaol species richness and Shannon diversity indexes of intestinal microbiota between yak and cattle calves at the 2nd week of their age. The 
social learning behavior of calves and the care of their mothers' to expose and feed fresh herbage

during early life promoted the successful colonization of intestine functional microbiota of yak calves, such as the main cellulolytic and hemicellulolytic bacteria (Fibrobacter, Prevotellaceae,

Clostridium, and Eubacterium), amylolytic bacteria (Streptococcus and Ruminobacter), proteolytic

Lactobacillus, and Bifidobacterium) [72-76, 79]. Diet is one of the main factors that influence the composition of intestinal microbiota, so an earlier intake of forage can promote the stability and healthy development of the intestinal microbiota of neonatal calves[64, 80-82]. Also, inherited hostassociated factors, such as genotype, gender, and immune status, might function as selective filters in the assembly process of the intestinal microbial community[83-85]. However, with the maturation and stabilization of calf intestinal microbiota, the difference between yak and cattle calves decreased gradually, which may be the result of a convergent evolution of the key genomic functions conserved between the hosts in interaction with the microbial partners [30, 86, 87]. functions in the hindgut, which provide energy to the host [88]. The higher predicted microbial energy metabolism at weeks 1 and 2 indicated that microbiota during early life tended to harvest more energy from the lumen substance for their growth and proliferation. Also, the significant increase in predicted amino acid metabolism of mucosa-attached bacteria at weeks 5 and 9 suggested that bacteria derived more energy from amino acid fermentation along with the increase of the age of calves. The observed temporal variations in predicted microbial functions of the hindgut bacteria suggested potential changes in energy harvesting mechanisms associated with host diets. It is noticeable that the functional prediction based on the 16S rRNA gene is biased and further 
metagenomic and metatranscriptomic studies are needed to assess the function of hindgut microbiota. However, the predicted function could provide preliminary information on the hindgut microbial functions of pre-weaned calves.

\section{Conclusions}

Gut microbes in early life are important for many aspects of animal immune [2, 89], metabolic [1], and neurobehavioral traits [90]. A central question is the extent to which microbiota composition is determined by the host genetics and environment. Most animal models can help identify heritable bacterial taxa and mechanisms, though the gut microbiota is shaped by multiple factors, and the relative contributions of host genetics and environments remain elusive. Therefore, in this study, we investigated the effect of maternal microbiota on the succession of intestinal microbiota in preweaning yak and cattle calves kept in the same natural pasture. Our results indicated that genetics and early maternal rearing had important effects on the maturation and stability of calf intestinal microbial community. We found that the intestinal microbiota of yak calves reached a relatively stable state earlier than those of cattle calves, following their long-term evolution and natural selection to better adapt to harsh environment on the QTP, as an old saying goes, "the neonate from the poor seems to grow up earlier". Noteworthy, our results revealed that under natural grazing conditions, the calves raised by their mothers could lick the maternal feces and eat fresh grass through the social learning behavior earlies, which might accelerate the establishment and relative stability of the normal intestinal functional microbiota. Therefore, our results suggest that living with mother for at least 1 week after birth may help to establish and stabilize the intestinal microbiota of the calve, and subsequently, lower economic loss caused by diarrhea of pre-weaning calves. 
584

\section{Additional file 1:}

Figure S1. Species diversity curse. A) Rarefaction curve, in which the abscissa is the number of sequences randomly drawn from a sample and the ordinate is the number of OTUs based on the number of sequences. B) Rank abundance, ordinal by OTUs abundance in abscissa and relative abundance in OTUs' relative abundance in ordinate. Different samples are represented by polylines of different colors.

Figure S2. Circos plot. (A) The microbial communities of different samples are phylogenetic at the genus level (top 100). (B and C) The average relative abundances of the most prevalent bacterial phyla and order in each sample type plotted for the samples from the maternal and calf fecal samples.

Figure S3. Bar plots showing differential abundant gut microbes of yak and cattle at A) 1, B) 2, C) 5, and D) 9 weeks before weaning, and E) their mothers, as identified by linear discriminant analysis (LDA) effect size (LEfSe). The bar plot shows scores for all the taxa with a LDA score $\geq 2$. Labels are shown at the family and genera levels.

\section{Additional file 2:}

Table S1. Comparison of alpha diversity of fecal microorganisms in different developmental stages of yak and cattle calves before weaning.

Table S2. The results of the Amova test.

Table S3. The relative abundance of the sample types between yak and cattle at the genus level (top $30)$. 
604

605

606

607

608

609

610

611

612

613

614

615

616

617

618

619

Table S4. The results of the PERMANOVA test based on Bray-Curtis distance.

Table S5. T-test of the intestinal microbial community at different developmental stages of yak and cattle at the genus level.

\section{Abbreviations}

QTP: The Qinghai-Tibetan Plateau; 16S rRNA: 16 Svedberg unit ribosomal ribonucleic acid; PCR:

Polymerase chain reaction; OTU: Operational taxonomic unit; NDMS: Non-metric multidimensional scaling; PCoA: Principal Coordinate Analysis; PERMANOVA: Permutational analysis of variance; SIMPER: Similarity percentage analysis.

\section{Acknowledgements}

The authors would like to thank Nana Yao and Peng Wang from Tianjin Novogene for help with some data analysis and programming in $\mathrm{R}$.

\section{Authors' contributions}

JBZ, PY, and XZD designed the study. JBZ, ZYL, RQ DK, MD, AAA, and SYW collected the samples. JBZ, ZYL, RQ KD, and MD performed bioinformatics and statistical analyses. JLH, SYW, GHS, RJL, PY, and XZD guided the data analysis and revised the manuscript. JBZ, ZYL, and XZD interpreted the data and wrote the manuscript. The authors read and approved the final manuscript.

\section{Funding}

This work was supported by a grant from the International Cooperation and Exchange Program of the National Natural Science Foundation of China (31461143020), the Innovation Program of Chinese Academy of Agricultural Sciences (00\&0656), and the Key Technologies Research \& 


\section{Availability of data and materials}

626

627

628

Raw sequencing data have been deposited at the NCBI Sequence Read Archive (SRA) under BioProject ID PRJNA688363.

\section{Ethics approval and consent to participate}

All experimental procedures in this study were approved by the Animal Care and Utilization Committee of Lanzhou Institute of Husbandry and Pharmaceutical Sciences of Chinese Academy of Agricultural Sciences.

\section{Consent for publication}

Not applicable

\section{Competing interest}

The authors declare that they have no competing interests.

\section{Author details}

${ }^{1}$ Key Laboratory of Yak Breeding Engineering, Lanzhou Institute of Husbandry and Pharmaceutical Sciences, Chinese Academy of Agricultural Sciences, Lanzhou 730050, China. ${ }^{2}$ Key Laboratory of Veterinary Pharmaceutical Development, Ministry of Agricultural and Rural Affairs, Lanzhou Institute of Husbandry and Pharmaceutical Sciences, Chinese Academy of Agricultural Sciences, Lanzhou 730050, China. ${ }^{3}$ Gannan Institute of Animal Husbandry Science, Hezuo 747000, China.

${ }^{4}$ Livestock Genetics Program, International Livestock Research Institute (ILRI), Nairobi 00100, Kenya. ${ }^{5}$ CAAS-ILRI Joint Laboratory on Livestock and Forage Genetic Resources, Institute of 
644 Animal Science, Chinese Academy of Agricultural Sciences (CAAS), Beijing 100193, China.

$645{ }^{6}$ School of Life Sciences, Lanzhou University, Lanzhou 730020, China. ${ }^{7}$ Department of Systems

646 Biology, Agricultural Biotechnology Research Institute of Iran, Agricultural Research, Education,

647 and Extension Organization, Karaj, Iran.

\section{$648 \quad$ References}

649 1. Tremaroli V, Backhed F. Functional interactions between the gut microbiota and host 650 metabolism. Nature. 2012;489(7415):242-9.

651

2. Hooper LV, Littman DR, Macpherson AJ. Interactions Between the Microbiota and the Immune System. Science. 2012;336(6086):1268-73.

653

3. Diaz Heijtz R, Wang S, Anuar F, Qian Y, Bjorkholm B, Samuelsson A, et al. Normal gut microbiota modulates brain development and behavior. Proc Natl Acad Sci U S A. 2011;108(7):3047-52.

4. Smits SA, Leach J, Sonnenburg ED, Gonzalez CG, Lichtman JS, Reid G, et al. Seasonal cycling in the gut microbiome of the Hadza hunter-gatherers of Tanzania. Science. 2017;357(6353):802-06.

5. de Goffau MC, Lager S, Sovio U, Gaccioli F, Cook E, Peacock SJ, et al. Human placenta has no microbiome but can contain potential pathogens. Nature. 2019;572(7769):329-34.

661 6. Sprockett D, Fukami T, Relman DA. Role of priority effects in the early-life assembly of the 662 gut microbiota. Nat Rev Gastroenterol Hepatol. 2018;15(4):197-205.

663 7. Furman O, Shenhav L, Sasson G, Kokou F, Honig H, Jacoby S, et al. Stochasticity constrained 664 by deterministic effects of diet and age drive rumen microbiome assembly dynamics. Nat Commun. 
666

667

668

669

670

671

672

673

674

675

676

677

678

679

680

681

682

683

684

685

686

687

8. Dominguez-Bello MG, Costello EK, Contreras M, Magris M, Hidalgo G, Fierer N, et al.

Delivery mode shapes the acquisition and structure of the initial microbiota across multiple body habitats in newborns. Proc Natl Acad Sci U S A. 2010;107(26):11971-5.

9. Rothschild D, Weissbrod O, Barkan E, Kurilshikov A, Korem T, Zeevi D, et al. Environment dominates over host genetics in shaping human gut microbiota. Nature. 2018;555(7695):210-15.

10. Chen CY, Chen CK, Chen YY, Fang A, Shaw GT, Hung CM, et al. Maternal gut microbes shape the early-life assembly of gut microbiota in passerine chicks via nests. Microbiome. 2020;8(1):129.

11. Liu JX, Taft DH, Maldonado-Gomez MX, Johnson D, Treiber ML, LemayQ DG, et al. The fecal resistome of dairy cattle is associated with diet during nursing. Nat Commun. 2019;10:1-15.

12. Gomez de Aguero M, Ganal-Vonarburg SC, Fuhrer T, Rupp S, Uchimura Y, Li H, et al. The maternal microbiota drives early postnatal innate immune development. Science. 2016;351(6279):1296-302.

13. Gensollen T, Iyer SS, Kasper DL, Blumberg RS. How colonization by microbiota in early life shapes the immune system. Science. 2016;352(6285):539-44.

14. Round JL, Mazmanian SK. The gut microbiota shapes intestinal immune responses during health and disease. Nat Rev Immunol. 2009;9(5):313-23.

15. Heinrichs AJ. Rumen development in the dairy calf. Adv Dairy Tech. 2005;17:179-87.

16. Castro JJ, Gomez A, White BA, Mangian HJ, Loften JR, Drackley JK. Changes in the intestinal bacterial community, short-chain fatty acid profile, and intestinal development of preweaned Holstein calves. 1. Effects of prebiotic supplementation depend on site and age. J Dairy Sci. 2016;99(12):9682-702. 
688

689

690

691

692

693

694

695

696

697

698

699

700

701

702

703

704

705

706

707

708

709

17. Hoover WH. Digestion and absorption in the hindgut of ruminants. J Anim Sci. 1978;46(6):1789-99.

18. Gressley TF, Hall MB, Armentano LE. Ruminant Nutrition Symposium: Productivity, digestion, and health responses to hindgut acidosis in ruminants. J Anim Sci. 2011;89(4):1120-30.

19. Alipour MJ, Jalanka J, Pessa-Morikawa T, Kokkonen T, Satokari R, Hynonen U, et al. Publisher Correction: The composition of the perinatal intestinal microbiota in cattle. Sci Rep. 2018;8(1):13792.

20. Yeoman CJ, Ishaq SL, Bichi E, Olivo SK, Lowe J, Aldridge BM. Biogeographical Differences in the Influence of Maternal Microbial Sources on the Early Successional Development of the Bovine Neonatal Gastrointestinal tract. Sci Rep. 2018;8(1):3197.

21. Gu Z, Zhao X, Li N, Wu C. Complete sequence of the yak (Bos grunniens) mitochondrial genome and its evolutionary relationship with other ruminants. Mol Phylogenet Evol. 2007;42(1):248-55.

22. Ding L, Long R, Shang Z, Wang C, Yang Y, Xu S. Feeding behaviour of yaks on spring, transitional, summer and winter pasture in the alpine region of the Qinghai-Tibetan plateau. Appl Anim Behav Sci. 2008;111(3-4):373-90.

23. Shao B, Long R, Ding Y, Wang J, Ding L, Wang H. Morphological adaptations of yak (Bos grunniens) tongue to the foraging environment of the Qinghai-Tibetan Plateau. J Anim Sci. 2010;88(8):2594-603.

24. Guan J, Long K, Ma J, Zhang J, He D, Jin L, et al. Comparative analysis of the microRNA transcriptome between yak and cattle provides insight into high-altitude adaptation. PeerJ. 2017;5:e3959. 
25. Zhou JW, Liu H, Zhong CL, Degen AA, Yang G, Zhang Y, et al. Apparent digestibility, rumen fermentation, digestive enzymes and urinary purine derivatives in yaks and Qaidam cattle offered forage-concentrate diets differing in nitrogen concentration. Livest Sci. 2018;208(10):14-21.

26. Ding XZ, Long RJ, Kreuzer M, Mi JD, Yang B. Methane emissions from yak (Bos grunniens) steers grazing or kept indoors and fed diets with varying forage concentrate ratio during the cold season on the Qinghai-Tibetan Plateau. Anim Feed Sci Tech. 2010;162(3-4):91-98.

27. Ahmad AA, Yang C, Zhang JB, Kalwar Q, Liang ZY, Li C, et al. Effects of Dietary Energy Levels on Rumen Fermentation, Microbial Diversity, and Feed Efficiency of Yaks (Bos grunniens). Front Microbiol. 2020;11:625.

28. Yang C, Ahmad AA, Bao PJ, Guo X, Wu XY, Liu JB, et al. Increasing dietary energy level improves growth performance and lipid metabolism through up-regulating lipogenic gene expression in yak (Bos grunniens). Anim Feed Sci Tech. 2020;263:114455.

29. Qiu Q, Zhang GJ, Ma T, Qian WB, Wang JY, Ye ZQ, et al. The yak genome and adaptation to life at high altitude. Nat Genet. 2012;44(8):946-49.

30. Zhang Z, Xu D, Wang L, Hao J, Wang J, Zhou X, et al. Convergent Evolution of Rumen Microbiomes in High-Altitude Mammals. Curr Biol. 2016;26(14):1873-9.

31. Guo W, Zhou M, Ma T, Bi S, Long R. Survey of rumen microbiota of domestic grazing yak during different growth stages revealed novel maturation patterns of four key microbial groups and their dynamic interactions. Animal Microbiome. 2020;2(1):23.

32. Klein-Jobstl D, Quijada NM, Dzieciol M, Feldbacher B, Wagner M, Drillich M, et al. Microbiota of newborn calves and their mothers reveals possible transfer routes for newborn calves' gastrointestinal microbiota. PLoS One. 2019;14(8):e0220554. 
33. Lima SF, Bicalho MLS, Bicalho RC. The Bos taurus maternal microbiome: Role in determining the progeny early-life upper respiratory tract microbiome and health. PLoS One. 2019;14(3):e0208014.

34. Fan P, Bian B, Teng L, Nelson CD, Driver J, Elzo MA, et al. Host genetic effects upon the early gut microbiota in a bovine model with graduated spectrum of genetic variation. ISME J. 2020;14(1):302-17.

35. Long RJ, Ding LM, Shang ZH, Guo XH. The yak grazing system on the Qinghai-Tibetan plateau and its status. Rangeland Journal. 2008;30(2):241-46.

36. Magoc T, Salzberg SL. FLASH: fast length adjustment of short reads to improve genome assemblies. Bioinformatics. 2011;27(21):2957-63.

37. Bokulich NA, Subramanian S, Faith JJ, Gevers D, Gordon JI, Knight R, et al. Quality-filtering vastly improves diversity estimates from Illumina amplicon sequencing. Nat Methods. 2013;10(1):57-9.

38. Caporaso JG, Kuczynski J, Stombaugh J, Bittinger K, Bushman FD, Costello EK, et al. QIIME allows analysis of high-throughput community sequencing data. Nat Methods. 2010;7(5):335-6.

39. Edgar RC. UPARSE: highly accurate OTU sequences from microbial amplicon reads. Nat Methods. 2013;10(10):996-8.

40. Quast C, Pruesse E, Yilmaz P, Gerken J, Schweer T, Yarza P, et al. The SILVA ribosomal RNA gene database project: improved data processing and web-based tools. Nucleic Acids Res. 2013;41(Database issue):D590-6.

41. Segata N, Izard J, Waldron L, Gevers D, Miropolsky L, Garrett WS, et al. Metagenomic biomarker discovery and explanation. Genome Biol. 2011;12(6):R60. 
42. Knights D, Kuczynski J, Charlson ES, Zaneveld J, Mozer MC, Collman RG, et al. Bayesian community-wide culture-independent microbial source tracking. Nat Methods. 2011;8(9):761-3.

43. Hammer O, Harper D, Ryan P. PAST: Paleontological Statistics Software Package for Education and Data Analysis. Palaeontol Electron. 2001;4:1-9.

44. Lex A, Gehlenborg N, Strobelt H, Vuillemot R, Pfister H. UpSet: Visualization of Intersecting Sets. IEEE Trans Vis Comput Graph. 2014;20(12):1983-92.

45. Langille MG, Zaneveld J, Caporaso JG, McDonald D, Knights D, Reyes JA, et al. Predictive functional profiling of microbial communities using $16 \mathrm{~S}$ rRNA marker gene sequences. Nat Biotechnol. 2013;31(9):814-21.

46. Roewer L, Kayser M, Dieltjes P, Nagy M, Bakker E, Krawczak M, et al. Analysis of molecular variance (AMOVA) of Y-chromosome-specific microsatellites in two closely related human populations. Hum Mol Genet. 1996;5(7):1029-33.

47. Georgios O, Vieira TAG, Carla F, Lucas BM, Silva MV, Carvalho BR, et al. Fecal Microbial Diversity in Pre-Weaned Dairy Calves as Described by Pyrosequencing of Metagenomic 16S rDNA. Associations of Faecalibacterium Species with Health and Growth. Plos One. 2013;8(4):e63157.

48. Matamoros S, Gras-Leguen C, Le Vacon F, Potel G, de La Cochetiere MF. Development of intestinal microbiota in infants and its impact on health. Trends Microbiol. 2013;21(4):167-73.

49. Malmuthuge N, Guan LL. Understanding host-microbial interactions in rumen: searching the best opportunity for microbiota manipulation. J Anim Sci Biotechnol. 2017;8(1):8.

50. Sonnenburg JL, Backhed F. Diet-microbiota interactions as moderators of human metabolism. Nature. 2016;535(7610):56-64.

51. Katri K, de Vos WM. Early life colonization of the human gut: microbes matter everywhere. 
Curr Opin Microbiol. 2018;44:70-78.

52. Castro JJ, Gomez A, White B, Loften JR, Drackley JK. Changes in the intestinal bacterial community, short-chain fatty acid profile, and intestinal development of preweaned Holstein calves.

2. Effects of gastrointestinal site and age. J Dairy Sci. 2016;99(12):9703-15.

53. Maslowski KM, Mackay CR. Diet, gut microbiota and immune responses. Nat Immunol. $2011 ; 12(1): 5-9$

54. Tung J, Barreiro LB, Burns MB, Grenier JC, Lynch J, Grieneisen LE, et al. Social networks predict gut microbiome composition in wild baboons. Elife. 2015;4:e05224.

55. Moeller AH, Foerster S, Wilson ML, Pusey AE, Hahn BH, Ochman H. Social behavior shapes the chimpanzee pan-microbiome. Sci Adv. 2016;2(1):e1500997.

56. Goffau M, Lager S, Sovio U, Gaccioli F, Cook E, Peacock S, et al. Human placenta has no microbiome but can contain potential pathogens. Nature. 2019;572.

57. Deng F, McClure M, Rorie R, Wang X, Chai J, Wei X, et al. The vaginal and fecal microbiomes are related to pregnancy status in beef heifers. J Anim Sci Biotechnol. 2019;10:92.

58. Dominguez-Bello MG, De Jesus-Laboy KM, Shen N, Cox LM, Amir A, Gonzalez A, et al. Partial restoration of the microbiota of cesarean-born infants via vaginal microbial transfer. Nat Med. $2016 ; 22(3): 250-3$

59. Colston TJ, Jackson CR. Microbiome evolution along divergent branches of the vertebrate tree of life: what is known and unknown. Mol Ecol. 2016;25(16):3776-800.

60. Abecia L, Ramos-Morales E, Martinez-Fernandez G, Arco A, Martin-Garcia AI, Newbold CJ, et al. Feeding management in early life influences microbial colonisation and fermentation in the rumen of newborn goat kids. Anim Prod Sci. 2014;54(9):1449-54. 
61. Galef BG, Jr., Giraldeau LA. Social influences on foraging in vertebrates: causal mechanisms and adaptive functions. Anim Behav. 2001;61(1):3-15.

62. De Paula Vieira A, von Keyserlingk MA, Weary DM. Presence of an older weaned companion influences feeding behavior and improves performance of dairy calves before and after weaning from milk. J Dairy Sci. 2012;95(6):3218-24.

63. Oikonomou G, Teixeira AG, Foditsch C, Bicalho ML, Machado VS, Bicalho RC. Fecal microbial diversity in pre-weaned dairy calves as described by pyrosequencing of metagenomic $16 \mathrm{~S}$ rDNA. Associations of Faecalibacterium species with health and growth. PLoS One. 2013;8(4):e63157.

64. Phillips CJ. The effects of forage provision and group size on the behavior of calves. J Dairy Sci. 2004;87(5):1380-8.

65. Soberon F, Raffrenato E, Everett RW, Van Amburgh ME. Preweaning milk replacer intake and effects on long-term productivity of dairy calves. J Dairy Sci. 2012;95(2):783-93.

66. Uetake K. Newborn calf welfare: a review focusing on mortality rates. Anim Sci J. 2013;84(2):101-5.

67. Borody TJ, Khoruts A. Fecal microbiota transplantation and emerging applications. Nat Rev Gastroenterol Hepatol. 2011;9(2):88-96.

68. Smits LP, Bouter KE, de Vos WM, Borody TJ, Nieuwdorp M. Therapeutic potential of fecal microbiota transplantation. Gastroenterology. 2013;145(5):946-53.

69. Aroniadis OC, Brandt LJ. Fecal microbiota transplantation: past, present and future. Curr Opin Gastroenterol. 2013;29(1):79-84.

70. Korpela K, Helve O, Kolho KL, Saisto T, Skogberg K, Dikareva E, et al. Maternal Fecal 
820

Microbiota Transplantation in Cesarean-Born Infants Rapidly Restores Normal Gut Microbial Development: A Proof-of-Concept Study. Cell. 2020;183(2):324-34.

71. Malmuthuge N, Griebel PJ, Guan le L. Taxonomic identification of commensal bacteria associated with the mucosa and digesta throughout the gastrointestinal tracts of preweaned calves. Appl Environ Microbiol. 2014;80(6):2021-8.

72. Palevich N, Kelly WJ, Ganesh S, Rakonjac J, Attwood GT. Butyrivibrio hungatei MB2003 Competes Effectively for Soluble Sugars Released by Butyrivibrio proteoclasticus B316(T) during Growth on Xylan or Pectin. Appl Environ Microbiol. 2019;85(3):e02056-18.

73. Ogata T, Makino H, Ishizuka N, Iwamoto E, Masaki T, Ikuta K, et al. Long-term high-grain diet altered the ruminal $\mathrm{pH}$, fermentation, and composition and functions of the rumen bacterial community, leading to enhanced lactic acid production in Japanese Black beef cattle during fattening. PLoS One. 2019;14(11):e0225448.

74. Drissi F, Merhej V, Angelakis E, El Kaoutari A, Carriere F, Henrissat B, et al. Comparative genomics analysis of Lactobacillus species associated with weight gain or weight protection. Nutr Diabetes. 2014;4(2):e109.

75. Han H, Xiao H, Zhang K, Lu Z. Impact of 4-epi-oxytetracycline on the gut microbiota and blood metabolomics of Wistar rats. Sci Rep. 2016;6:23141.

76. Quigley EM. Prebiotics and probiotics; modifying and mining the microbiota. Pharmacol Res. 2010;61(3):213-8.

77. Conroy ME, Shi HN, Walker WA. The long-term health effects of neonatal microbial flora. Curr Opin Allergy Clin Immunol. 2009;9(3):197-201.

78. Backhed F, Roswall J, Peng Y, Feng Q, Jia H, Kovatcheva-Datchary P, et al. Dynamics and 
842

843

844

845

846

847

848

849

850

851

852

853

854

855

856

857

858

859

860

861

862

863

Stabilization of the Human Gut Microbiome during the First Year of Life. Cell Host Microbe. 2015;17(5):690-703.

79. Abe F, Ishibashi N, Shimamura S. Effect of Administration of Bifidobacteria and Lactic Acid Bacteria to Newborn Calves and Piglets. J Dairy Sci. 1995;78(12):2838-46.

80. Rey M, Enjalbert F, Combes S, Cauquil L, Bouchez O, Monteils V. Establishment of ruminal bacterial community in dairy calves from birth to weaning is sequential. J Appl Microbiol. 2014;116(2):245-57.

81. Jami E, Israel A, Kotser A, Mizrahi I. Exploring the bovine rumen bacterial community from birth to adulthood. ISME J. 2013;7(6):1069-79.

82. Vieira AD, von Keyserlingk MAG, Weary DM. Presence of an older weaned companion influences feeding behavior and improves performance of dairy calves before and after weaning from milk. J Dairy Sci. 2012;95(6):3218-24.

83. Bevins CL, Salzman NH. The potter's wheel: the host's role in sculpting its microbiota. Cell Mol Life Sci. 2011;68(22):3675-85.

84. Bolnick DI, Snowberg LK, Hirsch PE, Lauber CL, Org E, Parks B, et al. Individual diet has sex-dependent effects on vertebrate gut microbiota. Nat Commun. 2014;5:4500.

85. Benson AK, Kelly SA, Legge R, Ma F, Low SJ, Kim J, et al. Individuality in gut microbiota composition is a complex polygenic trait shaped by multiple environmental and host genetic factors.

Proc Natl Acad Sci U S A. 2010;107(44):18933-8.

86. Maire J, Vincent-Monegat C, Masson F, Zaidman-Remy A, Heddi A. An IMD-like pathway mediates both endosymbiont control and host immunity in the cereal weevil Sitophilus spp. Microbiome. 2018;6(1):6. 

on convergent evolution of Shigella species. PLoS Genet. 2020;16(7):e1008931.

866 88. McNeil NI. The contribution of the large intestine to energy supplies in man. Am J Clin Nutr. $867 \quad 1984 ; 39(2): 338-42$. Rev. 2010;90(3):859-904.

871 microbiota-gut-brain axis: neurobehavioral correlates, health and sociality. Front Integr Neurosci. $872 \quad 2013 ; 7: 70$. 


\section{A Sampling Area}
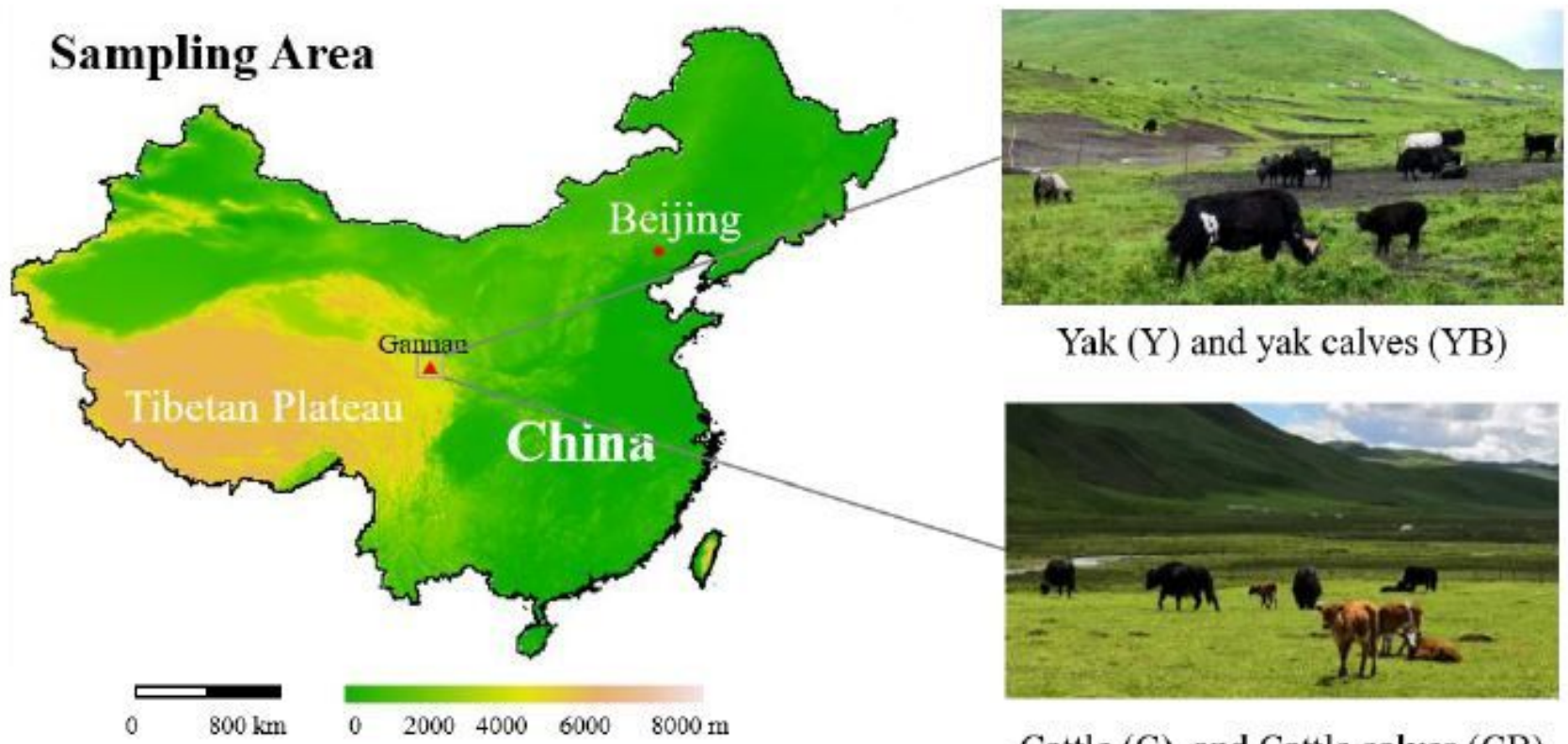

Yak (Y) and yak calves (YB)

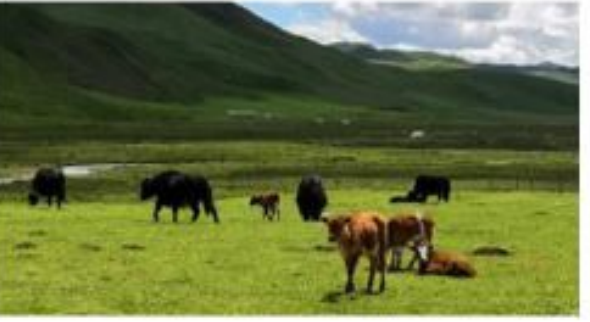

Cattle (C) and Cattle calves (CB)

\section{B Samples Types}

Maternal Microbiota

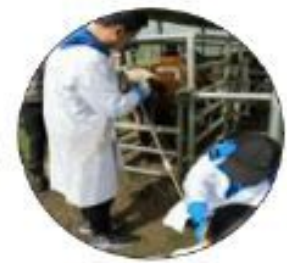

Rumen fluid

$\mathrm{YR}, \mathrm{n}=8$

$\mathrm{CR}, \mathrm{n}=8$

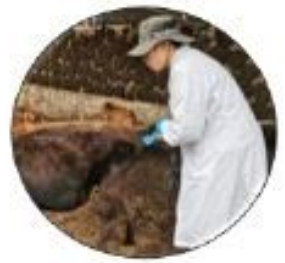

Feces

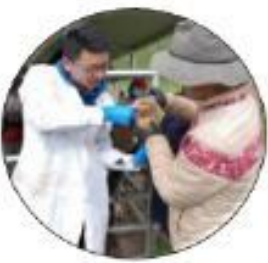

Oral cavity

YOc, $\mathrm{n}=8$

$\mathrm{COc}, \mathrm{n}=8$
$\mathrm{CF}, \mathrm{n}=8$

\section{WI}

Feces

YBWIF, $\mathrm{n}=5$

CBWIF, $\mathrm{n}=6$

\section{W2}

Feces

YBW2F, $\mathrm{n}=6$

CBW2F, $\mathbf{n}=7$

\section{W5}

Feces

YBW5F, $\mathrm{n}=6$

CBW5F, $\mathrm{n}=9$

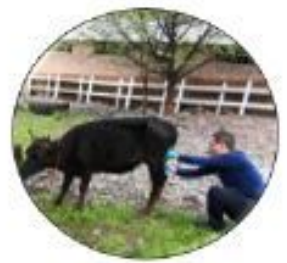

Breast skin

YBs, $\mathrm{n}=8$

CBs, $n=8$

Pre-weaning

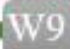

Feces

YBW9F, $\mathrm{n}=7$

CBW9F, $\mathrm{n}=7$

\section{Figure 1}

Intestinal microbial source and succession analysis of yak (YB) and cattle (CB) calves on the same pasture at different weeks during pre-weaning. Note: The designations employed and the presentation of the material on this map do not imply the expression of any opinion whatsoever on the part of Research Square concerning the legal status of any country, territory, city or area or of its authorities, or concerning the delimitation of its frontiers or boundaries. This map has been provided by the authors. 


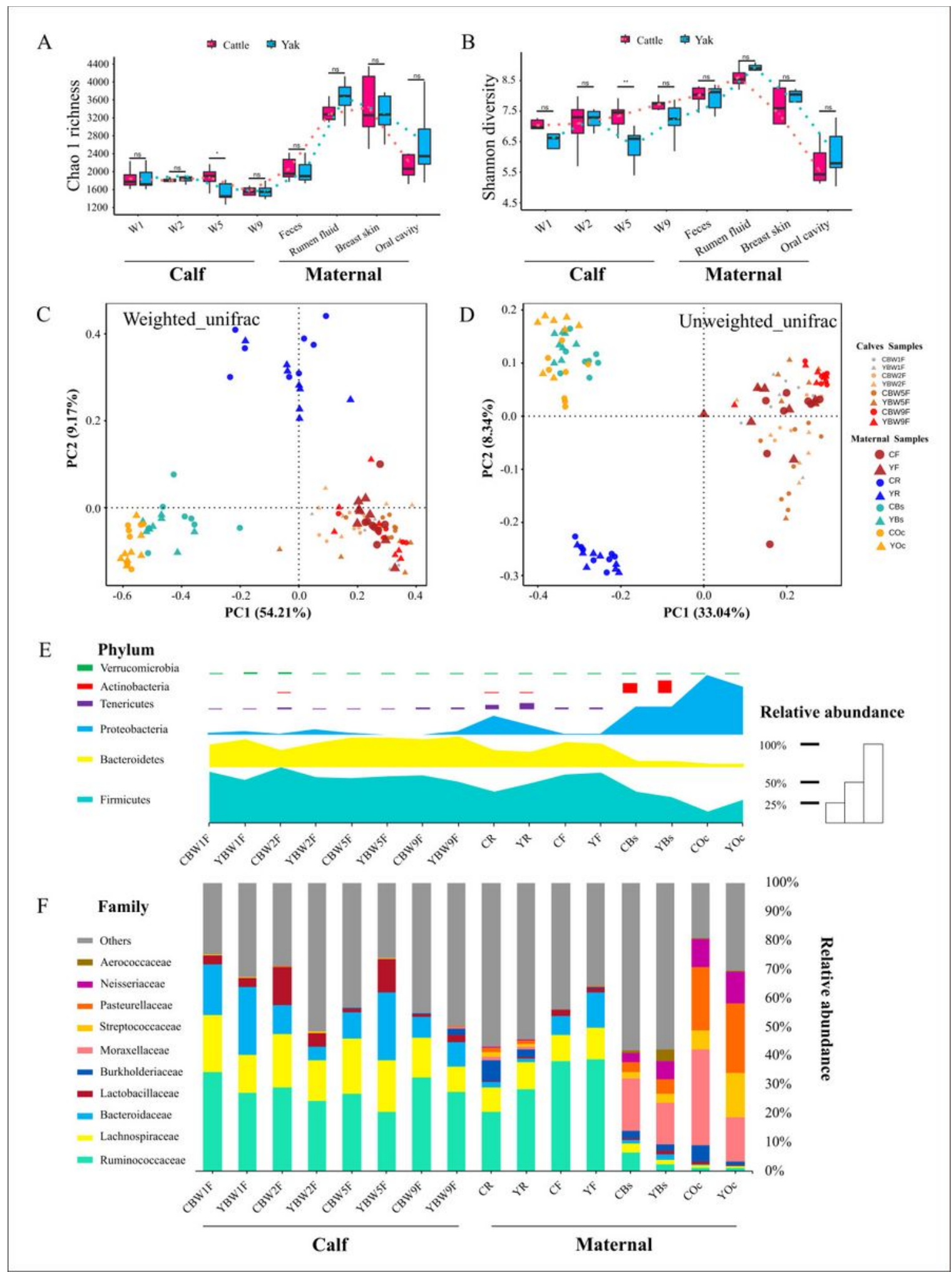

Figure 2

Compositions of the calf and maternal microbiota. (A) The Chao1 species richness and (B) Shannon diversity indexes of microbial communities in the samples of yak and cattle calf feces (at 1,2, 5, and 9 weeks of their ages), and their mothers (rumen fluid, feces, oral cavity, and breast skin). Distances between the samples, based on OUT similarity (OTU similarity $\geq 97 \%$ ) calculated using (C) weighted UniFrac distances in all samples; and (D) unweighted UniFrac distances in all samples; both visualized in 
PCoA plots. A greater distance between two points infers a lower similarity, whereas similar OTUs cluster together. $(E)$ The average relative abundances of the most prevalent bacterial phyla (bar length and area plot) in each sample type are plotted for samples from the maternal microbiota and calf feces. (F) Bar plots depict the relative abundances of bacterial families from the maternal samples and calf feces; unclassified taxa and bacterial families which have a relative abundance less than $1 \%$ were grouped into "Others".

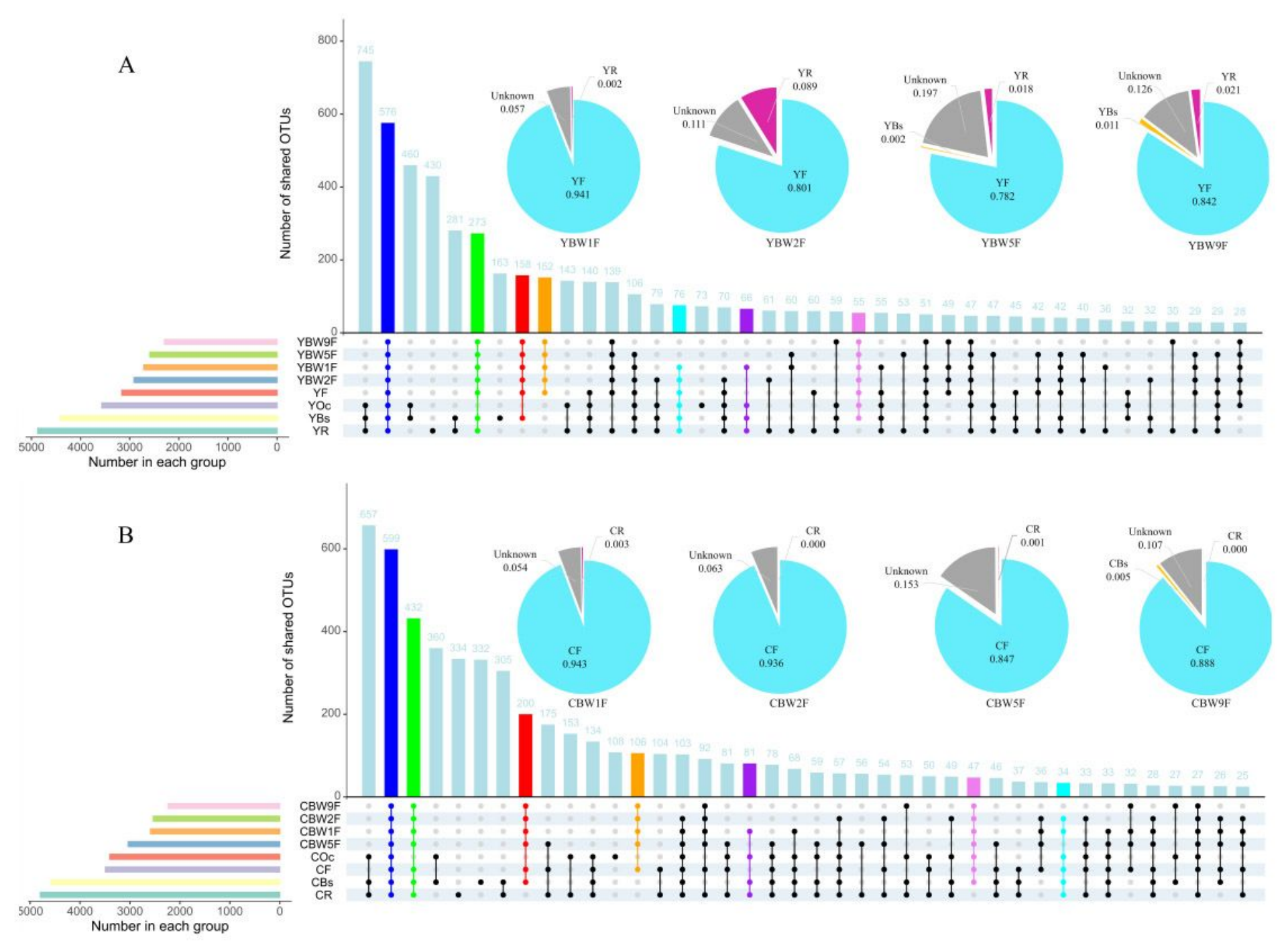

\section{Figure 3}

Community-level modeling and source-tracking analyses of the maternal sources in early calf gut community assembly. Yak (A) and cattle (B) animals are displayed separately. UpSet plots of common OTUs in the samples from the calf feces (at 1,2, 5, and 9 weeks of their ages) and their mothers (rumen fluid, feces, oral cavity, and breast skin). Only OTUs with an overall abundance across all samples greater than $0.01 \%$ are included, the 40 intersections which involved the greatest number of OTUs are displayed. The pie charts show the predicted proportions of sequences in the gut microbiota of both yak and cattle calves at different weeks pre-weaning $(1,2,5$ and 9 weeks) that originated from their maternal microbial communities (rumen fluid, feces, oral cavity, and breast skin). 


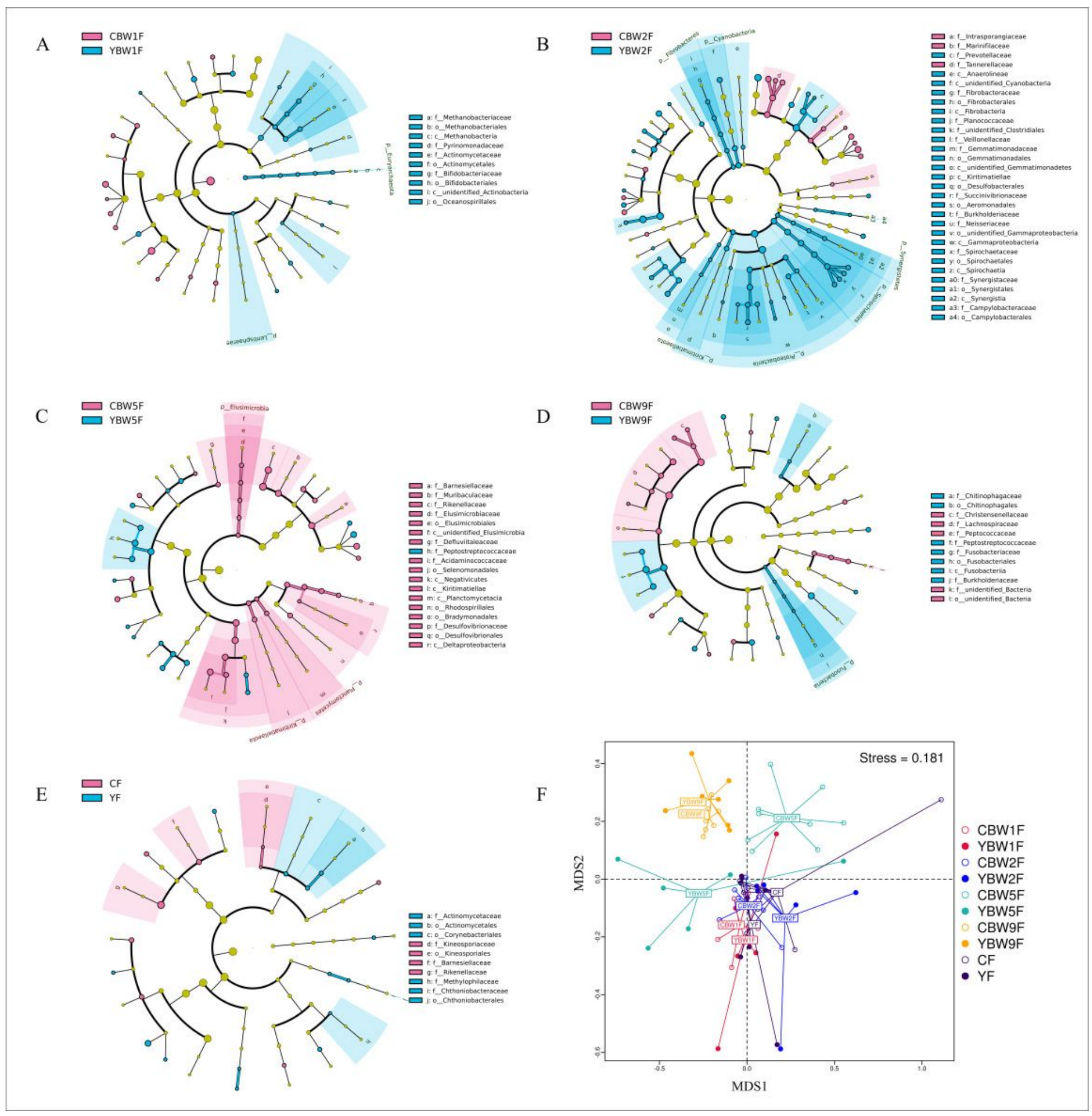

\section{Figure 4}

LEfSe analysis. The cladograms indicate the phylogenetic distribution of the intestinal microbiota of yak and cattle at (A) 1, (B) 2, (C) 5, and (D) 9 weeks before weaning and their mothers (E) using the Linear Discriminant Analysis (LDA) Effect Size (LEfSe) method. Differences are represented by treatment colors (nattier blue indicates yak, pink indicates cattle, and yellow non-significant). Circle's diameters are proportional to the taxon's abundances. Circles represent taxonomic ranks from domain to species from inside to out layers. The LDA cut-off score is 2 . Letters in front of OTUs represent taxonomic levels ( $p$, 
phylum; c, class; o, order; f, family). (F) The gut microbial community structures across calf and maternal samples. Non-metric multidimensional scaling (NMDS) ordination based on Bray-Curtis distances among sample types are plotted based on OTU abundances in the calf and maternal samples.

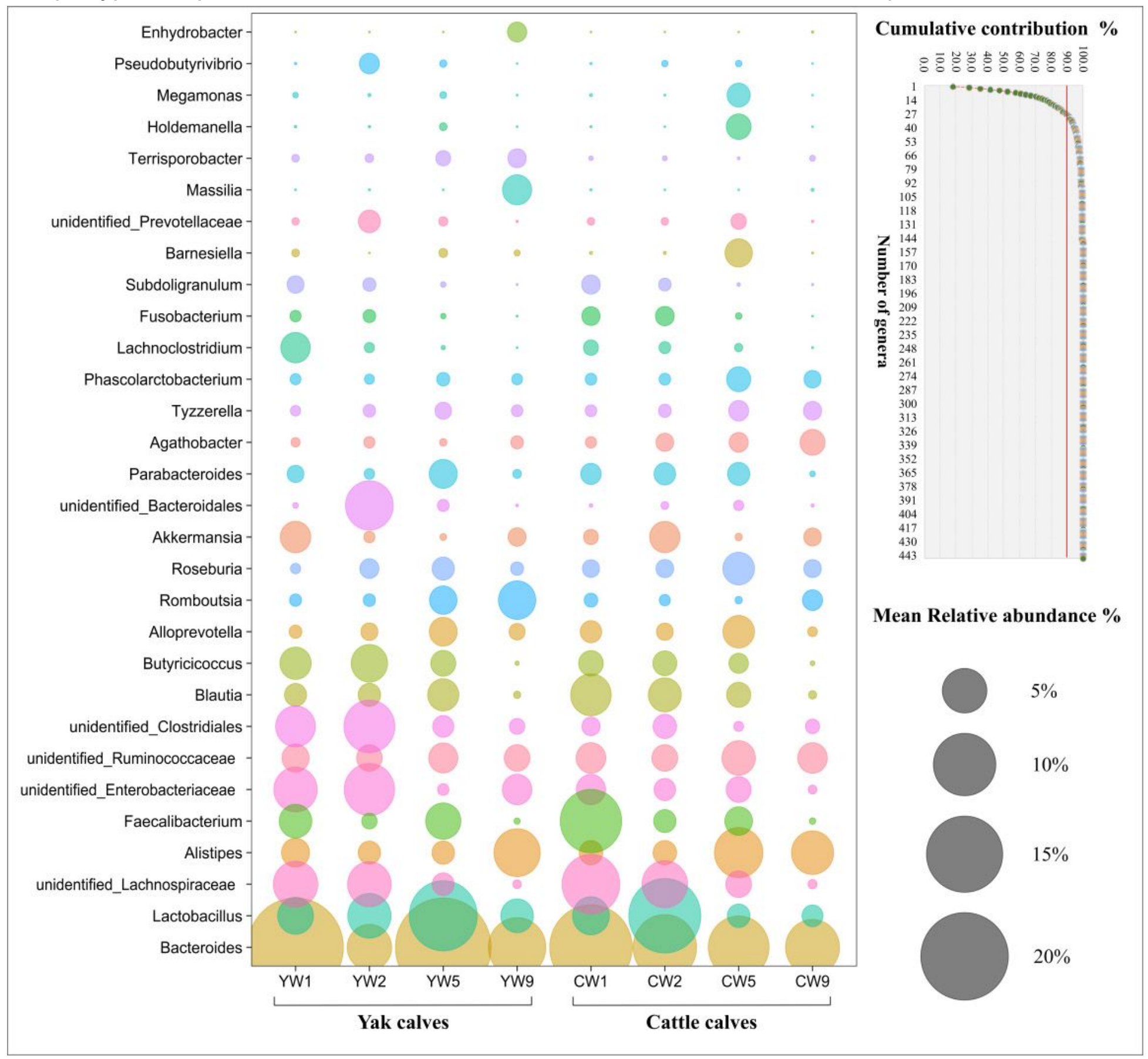

\section{Figure 5}

Similarity percentage analysis (SIMPER). The line graph shows the result of the SIMPER analysis performed with the PAST program, where all genera under a defined threshold $(90 \%)$ of the cumulative contribution are declared as specialized genera. Bubble-plot represents specialized microbial communities in the intestines of yak and cattle calves before weaning (at 1,2, 5, and 9 weeks of their ages), while the size of the bubble represents the relative abundance of the respective genus. 


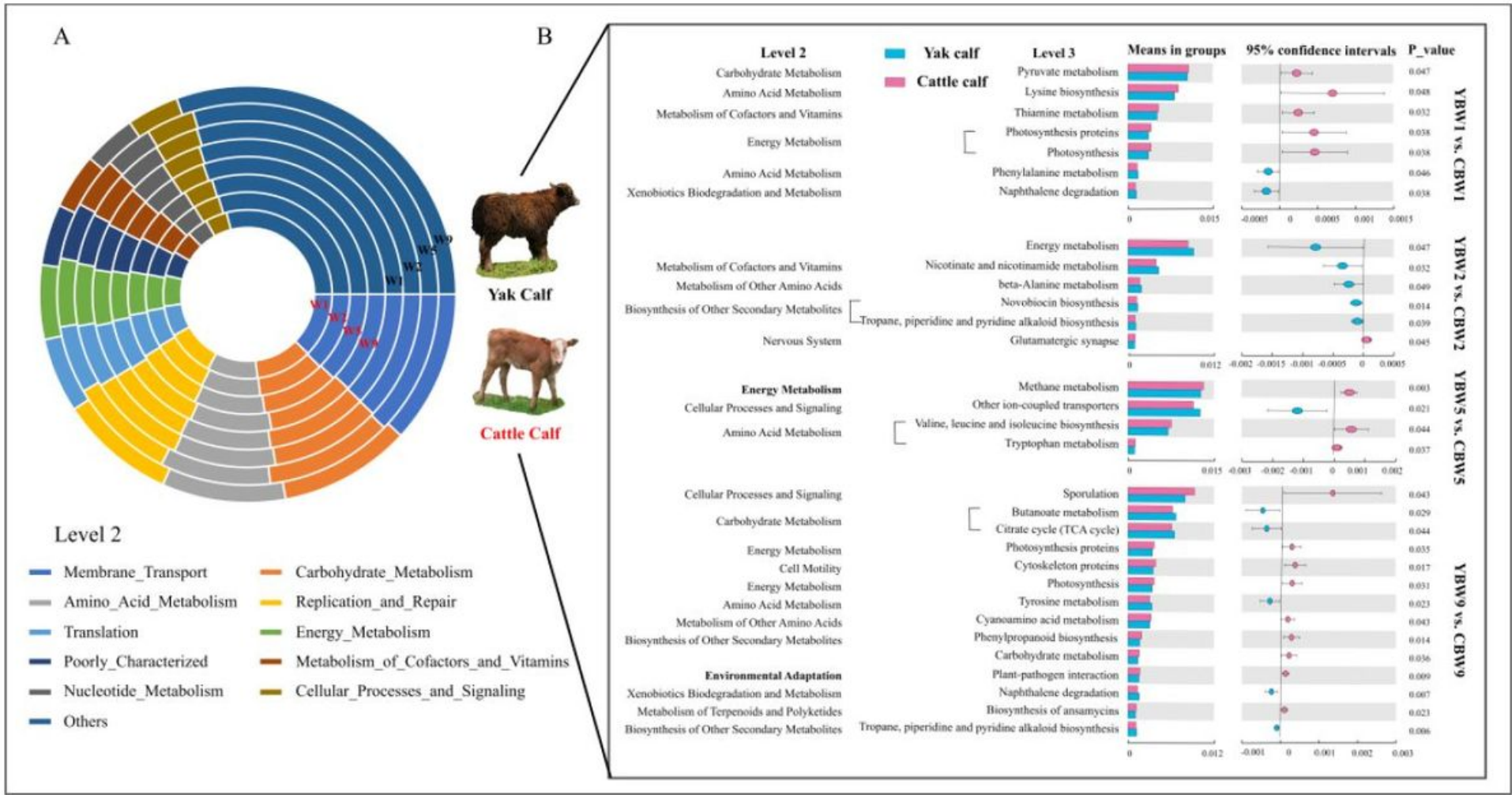

\section{Figure 6}

Predicted microbial functions using PICRUSt. (A) Relative abundances of level 2 KEGG pathways are described by the age of calves within different species (different weeks before weaning) in circular bar plots. (B) The comparison of the function of intestinal microbiota at different stages of development before weaning between yak and cattle calves based on KEGG level 3 annotation.

\section{Supplementary Files}

This is a list of supplementary files associated with this preprint. Click to download.

- Additionalfile1.pdf

- Additionalfile2.xlsx

- FigureS1.pdf

- FigureS2.pdf

- FigureS3.pdf 